\title{
A Review of Indocyanine Green Fluorescent Imaging in Surgery
}

\author{
Jarmo T. Alander, ${ }^{1}$ Ilkka Kaartinen, ${ }^{2}$ Aki Laakso, ${ }^{3}$ Tommi Pätilä, ${ }^{4}$ \\ Thomas Spillmann, ${ }^{5}$ Valery V. Tuchin, ${ }^{6,7,8}$ Maarit Venermo, ${ }^{9}$ and Petri Välisuo ${ }^{1}$ \\ ${ }^{1}$ Department of Electrical Engineering and Energy Technology, University of Vaasa, Vaasa, Finland \\ ${ }^{2}$ Department of Hand Surgery, Tampere University Hospital, 33680 Tampere, Finland \\ ${ }^{3}$ Department of Neurosurgery, Helsinki University Central Hospital (HUCH), Helsinki, Finland \\ ${ }^{4}$ Department of Cardiosurgery, Helsinki University Central Hospital, Helsinki, Finland \\ ${ }^{5}$ Department of Equine and Small Animal Medicine, University of Helsinki, Helsinki, Finland \\ ${ }^{6}$ Saratov State University, Saratov 410012, Russia \\ ${ }^{7}$ Institute of Precise Mechanics and Control, Russian Academy of Sciences, Saratov 410028, Russia \\ ${ }^{8}$ University of Oulu, Oulu, Finland \\ ${ }^{9}$ Clinic of Angiosurgery, Helsinki University Central Hospital, Helsinki, Finland
}

Correspondence should be addressed to Jarmo T. Alander, jarmo.alander@uwasa.fi

Received 1 September 2011; Accepted 1 February 2012

Academic Editor: Guowei Wei

Copyright ( $) 2012$ Jarmo T. Alander et al. This is an open access article distributed under the Creative Commons Attribution License, which permits unrestricted use, distribution, and reproduction in any medium, provided the original work is properly cited.

The purpose of this paper is to give an overview of the recent surgical intraoperational applications of indocyanine green fluorescence imaging methods, the basics of the technology, and instrumentation used. Well over 200 papers describing this technique in clinical setting are reviewed. In addition to the surgical applications, other recent medical applications of ICG are briefly examined.

\section{Introduction}

Fluorescence Imaging (FI) is one of the most popular imaging modes in biomedical sciences for the visualisation of cells and tissues both in vitro and in vivo [1]. The benefits of FI include

(i) high contrast, that is, signal to noise ratio (SNR): only the target, not background, is visible because separate wavelengths are used for illumination and recording (cf. Figure 4);

(ii) high sensitivity: extremely small concentrations can often be made visible;

(iii) Gives molecular information: makes some (bio) chemistry spatially and temporally visible;

(iv) great tools for research: several possible imaging modes, most of which are unique;

(v) cheap: the optical instrumentation and computing needed are quite simple;

(vi) easy to use: resembles classical staining.
Fluorescent imaging is a relatively recent imaging method and thus still developing in many ways. This is especially true for indocyanine green (ICG) imaging in its new clinical applications recently proposed in various branches of surgical medicine, although it has been used in some clinical applications routinely already for almost sixty years. Thus, ICG is well known in its established clinical applications, which greatly facilitates its introduction to new applications. From an engineering point of view, image and video processing seems to be among the main areas in which ICG imaging (ICGI) has potential for major developments, for example, for analysis of ICG fluorescence dynamics [2] (cf. Figure 2). This means, among other things, that a lot of computing development work is still needed for a broader acceptance of various emerging ICG-based medical imaging methods [3].

1.1. Indocyanine Green Angiography. Indocyanine green has been used for decades in ophthalmology for imaging retinal blood vessels, that is, in retinal angiography. However, fluorescein operating in visual wavelengths has been much more 


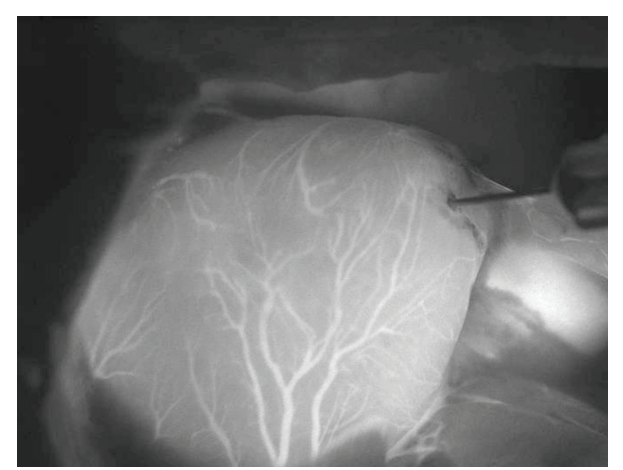

FIgURE 1: A typical ICGA image: heart of a rat. Coronary arteries clearly visible. Liver shining on the right. Magnification $20 \times$. Image taken by Dr. Outi Villet at HUCH by our prototype microscope device shown in Figure 6.

popular in retinal angiography partly because it is visible without any electronic cameras. However, the objects of imaging, retinal layers, with fluorescein and ICG somewhat differ. ICG gives information about deeper lying blood veins because it operates in near infrared (NIR), in which tissues are much more translucent than in visual wavelengths.

The principle of fluorescence imaging used in ICG angiography (ICGA) is simple: illuminate the tissue of interest with light at the excitation wavelength (about 750 to $800 \mathrm{~nm}$ ) while observing it at longer emission wavelengths (over $800 \mathrm{~nm}$; Figure 4). To create a simple ICGA device, only a couple of filters are needed in addition to a proper camera and a light source, which can be quite small and suitable even for portable use [4]. The filters are needed to prevent the mixing of the excitation (strong) and fluorescing (weak) rays to sum at the sensor. Even if the fluorescence is only a small fraction of the excitation intensity (Table 9: row 1 versus row 10), a surprisingly good signal to noise ratio (SNR) is attained: a brightly fluorescing object, mostly blood vessels containing ICG, can be clearly seen on an almost black background (see Figure 1). Without the filters, the weak fluorescence image cannot be seen among the strong reflection of the excitation light.

Indocyanine green dye was developed for near-infrared (NIR) photography by the Kodak Research Laboratories in 1955 and was approved for clinical use already in $1956[6,7]$. However, it took over ten years before ICG was used for angiography [8]. For retinal angiography it has been used from early $70 \mathrm{~s}[9]$.

1.2. Related Work. A few reviews of ICG and ICGA have been published. Those are briefly reviewed in what follows. Frangioni gives a review on in vivo fluorescent imaging including ICG-assisted imaging [10]. Amiot et al. give a review of the different NIR fluorescent materials developed and proposed for biomedical imaging [11]. Choyke et al. give a review of the toxicity of organic fluorophores including ICG used in molecular imaging [12]. For a recent review of ICG in retinal angiography, see for example, [13-15]. ICG, and infracyanine green in macular hole surgery are
TABLe 1: The number of ICG-related publications: queries from databases PubMed, ISI, SPIE, and IEEE (26.7.2011).

\begin{tabular}{lcccc}
\hline Keyword & PubMed & ISI & SPIE & IEEE \\
\hline "Indocyanine" (ICG) & 6069 & 5159 & 301 & 57 \\
ICG and "surgery" & 2160 & 1059 & 25 & 4 \\
ICG and "liver" & 2031 & 1164 & 18 & 5 \\
ICG and "retina" & 1176 & 406 & 7 & 11 \\
ICG and "cancer" & 816 & 372 & 49 & 14 \\
ICG and "tomography" & 748 & 594 & 29 & 10 \\
ICG and "imaging" & 697 & 828 & 130 & 43 \\
ICG and "heart" & 483 & 174 & 3 & 0 \\
ICG and "wound" & 190 & 53 & 24 & 1 \\
ICG and "lymph" & 128 & 115 & 11 & 1 \\
ICG and "brain" & 127 & 119 & 16 & 0 \\
ICG and "breast" & 105 & 189 & 35 & 10 \\
ICG and "laparoscopy" & 47 & 26 & 0 & 0 \\
\hline
\end{tabular}

reviewed in [16]. ICG and some similar dyes in vitreoretinal surgery are reviewed in [17]. A short overview of early works on fluorescence-enhanced contrast imaging and tomography is given in [18]. A recent review of ICG in assessment of liver function is given in [19], and a personal history view on ICG in liver monitoring is given by Paumgartner [20]. Houston gives an overview of in vivo small animal studies on fluorescent contrast agents [21]. te Velde et al. have recently briefly reviewed all papers regarding fluorescent dyes in surgical oncology [22], Schaafsma et al. have reviewed ICG in oncologic surgery [23], Polom et al. ICG usage in oncology and especially in sentinel lymph node biopsy (SLNB) [24] and Luo et al. NIR dyes including ICG in cancer targeting and imaging [25]. In a recent review Kaiser et al. review optical methods, including ICG imaging, in noninvasive assessment of burn wound severity [26]. An excellent and illustrative review of ICG in clinical imaging of the lymphatic system is given recently by Marshall et al. [27]. National Library of Medicine maintains a database of contrast agents called MICAD [28].

1.3. ICG Publications. To get an idea of the volume of ICGrelated research activities, the number of ICG-related publications in several databases (PubMed, ISI, IEEE, and SPIE) was collected in Table 1 and classified according to the main application areas. As anticipated, most research on ICG seems to be related to clinical sciences and not to, for example, engineering, optics, spectroscopy, or imaging, which indicates that there is still much work left to reveal all the technical potential of ICG. For instance, most of the works on image processing deal with ICGA of the retina only. On the other hand, the long and routine use of ICG in some clinical applications, such as retinal imaging, has provided us with much invaluable knowledge and experience useful in the development of new clinical applications, which are anticipated to be introduced exceptionally swiftly and at the same time at both relatively low risk and cost. 


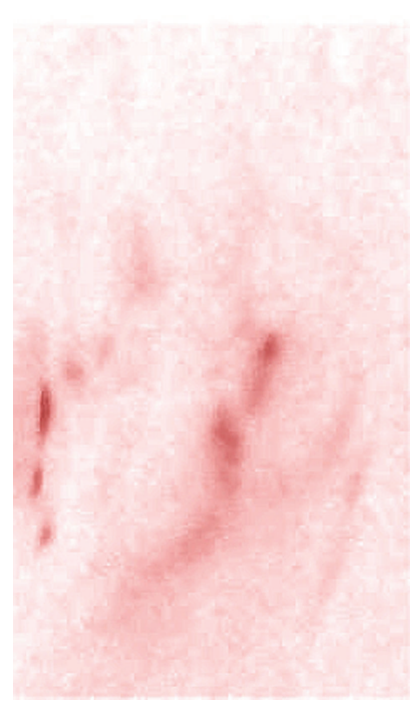

(a)

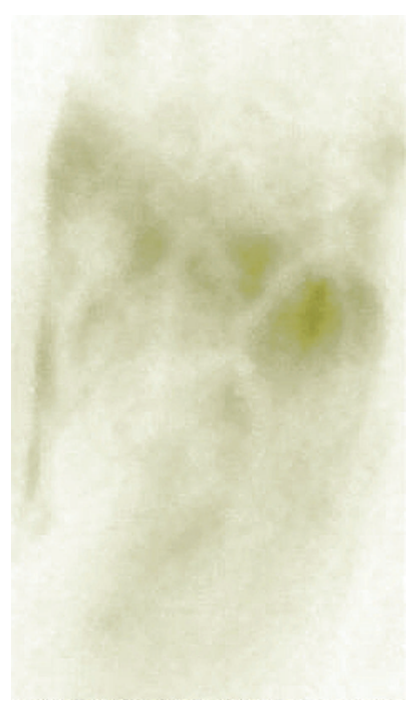

(b)

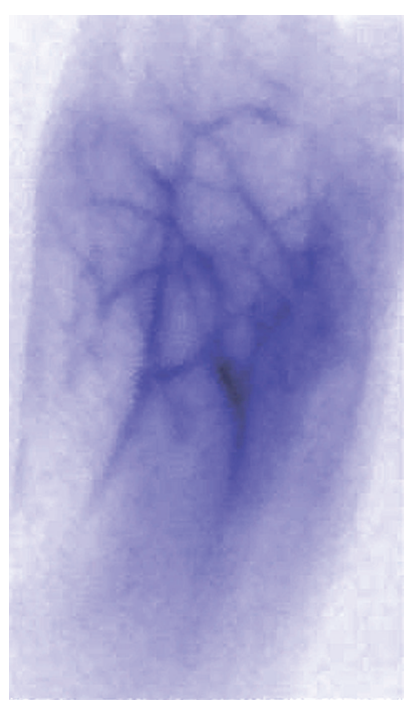

(c)

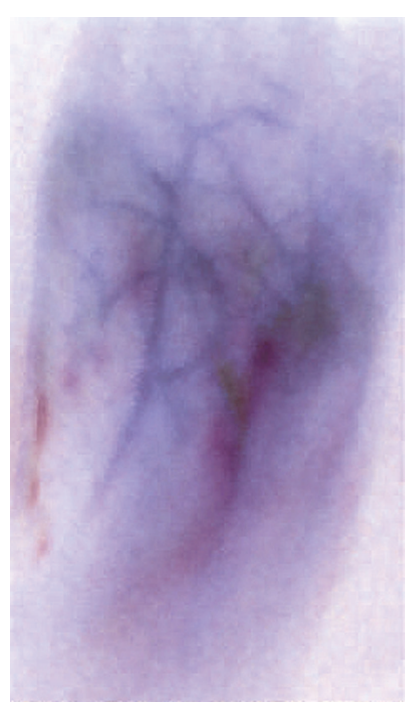

(d)

FIGURE 2: Simple image processing and pseudocoloring: ICG-VA frames of a leg (toes up) after the injection of ICG: (a) at about $30 \mathrm{~s}$ showing deep lying arteries in red, (b) at about 60 s showing mainly capillaries in yellowish green, (c) at about 90 s showing mainly subcutaneous veins in blue, and (d) fusion of the first three images. Image processing steps: negative of the original image and some intensity remapping. Fusion by using CMYC model. For more information see [5]. The original B/W images were taken with PDE by Dr. Hiroaki Terasaki (visiting HUCH from Tokyo Medical and Dental University Hospital of Medicine) and later processed by one of the authors (P. Välisuo).

The number of annual ICG publications according to PubMed is given in Table 2. The increase of publications has been about 10 papers per year. This number is expected to increase due to the emerging clinical applications described later in this paper. We can already (Fall 2011) see a considerable increase of papers for the years 2009 and 2010. According to Espacenet (6.5.2010), there are over 170 ICGrelated patents.

\section{Properties of Indocyanine Green}

The principal advantages causing the rapid acceptance of ICG were the presence of the absorption maximum, around $800 \mathrm{~nm}$, the confinement to the vascular compartment through binding with plasma proteins, the low toxicity $\left(\mathrm{LD}_{50}\right.$ of $50-80 \mathrm{mg} / \mathrm{kg}$ for animals http://www.drugs.com/pro/ indocyanine-green.html), and the rapid excretion, almost exclusively into the bile.

ICG fluoresces at about $800 \mathrm{~nm}$ and longer wavelengths. The exact shape of the spectra depends somewhat on the chemical environment and physical condition of ICG molecules like temperature and ICG concentration. The spectra are also smoothly varying, thus the exact wavelength values given in the literature somewhat vary depending also on the excitation light spectra and the filters used. Table 5 gives some excitation and observation wavelengths used in different ICG imaging instruments. The sensitivity of fluorescence spectra on molecular environment means that ICG is a potential molecular probe [29]. This has not yet been used in clinical applications. This is obviously one potential direction of ICG imaging development. Related to this direction is the need for better understanding of the
TABle 2: From PubMed (2.11.2009) "Indocyanine": (years 20072010: 17.8.2011).

\begin{tabular}{|c|c|c|c|}
\hline \multicolumn{2}{|c|}{ Recent } & \multicolumn{2}{|c|}{ Early } \\
\hline Year & Number & Year & Number \\
\hline 2010 & 397 & 1970 & 38 \\
\hline 2009 & 369 & 1969 & 35 \\
\hline 2008 & 295 & 1968 & 29 \\
\hline 2007 & 275 & 1967 & 37 \\
\hline 2006 & 274 & 1966 & 25 \\
\hline 2005 & 277 & 1965 & 20 \\
\hline 2004 & 295 & 1964 & 16 \\
\hline 2003 & 295 & 1963 & 8 \\
\hline 2002 & 240 & 1962 & 5 \\
\hline 2001 & 219 & 1961 & 2 \\
\hline 2000 & 224 & 1960 & 10 \\
\hline 1999 & 195 & 1959 & 0 \\
\hline- & - & 1958 & 1 \\
\hline 1989 & 96 & 1957 & 0 \\
\hline- & - & 1956 & 0 \\
\hline 1979 & 69 & 1955 & 0 \\
\hline- & - & & \\
\hline
\end{tabular}

binding of ICG molecules in different cells and tissues. This is clearly an arena for some further systematic basic research using fluorescence microscopy that may later possibly even lead to some major imaging innovations in biomedical applications. 
ICG has several clinically excellent properties, which has been thoroughly verified during its long clinical use:

(i) patient safety: nontoxic and nonionizing,

(ii) ideal for angiography: binds efficiently to blood lipoproteins, that is, it does not leak from circulation,

(iii) short life time in blood circulation allowing repeated applications,

(iv) good SNR: there is not much NIR autofluorescence in tissue giving low noise background,

(v) deep imaging: operates in tissue optical window (NIR), and

(vi) simple and cheap imaging devices (Hamamatsu: [30]).

What is so new in ICG angiography? Recently new successful medical applications, mainly in surgery, have been introduced. Some of the ICG's subexcellent properties provide further challenges to research and engineering development:

(i) ICG is very recent in many applications such as cancer treatment, reconstructive surgery, and even in cholecystectomy,

(ii) ICG needs some NIR imaging device to be visible,

(iii) for some applications ICG seems to need online illumination control facility,

(iv) clinically usable chemical derivatives for more specific physicochemical imaging do not yet exist,

(v) ICG injection solution contains some sodium iodide; thus, an allergic reaction is possible,

(vi) ICG is unstable in solutions $(10 \mathrm{~h})$ and when exposed to light, and

(vii) ICG has nonlinear fluorescence quantum yield versus concentration.

The development work for creating even better NIR contrast agents is going on in a few laboratories. Some of the proposed new molecules are based on ICG, while there are also totally different approaches such as quantum dot-based contrast agents [31,32].

2.1. Structure and Stability. Indocyanine green is a tricarbocyanine dye having a molecular weight of $751.4 \mathrm{Da}$. It is a negatively charged ion that belongs to the large family of cyanine dyes [33]. Dry ICG is stable at room temperature. This is also the form of pharmaceutically available ICG. ICG is soluble in water $(1 \mathrm{mg} / \mathrm{mL})$ but is not readily soluble in saline. Therefore, ICG should first be dissolved in water and only after this diluted with saline if an isotonic solution is needed (Sigma-Aldrich). Some chemicals, such as sodium polyaspartate (PASP), can be used to stabilise ICG in water and blood solutions, for example, when blood samples should be stored for several days. The use of PASP has been demonstrated also in vivo for a rat model $[34,35]$. The chemical decomposition of ICG can be inhibited by sodium azide $\mathrm{NaN}_{3}$, a quencher of singlet oxygen, that is, an antioxidant [36]. Also storage of the ICG solution at low temperature $\left(4^{\circ} \mathrm{C}\right)$ inhibits decomposition, while storage at room temperature facilitates decomposition [7].

2.1.1. Spectral Stability. In aqueous solutions, ICG molecules tend to aggregate, which influences their optical properties [37]. The aggregation depends on concentration and time; thus, ICG solutions do not follow Lambert-Beer's law above $15 \mathrm{mg} / \mathrm{L}$ in plasma [38]. The spectral stabilisation is fastest when ICG is dissolved in distilled water, and thus Landsman et al. do not recommend adding isotonic saline and/or albumin to the injectate, when fast spectral stability is essential, for example, when using ICG for quantitative purposes [38]. In tissues and cells the NIR absorption peak, due to binding with cell proteins, is moved to longer wavelengths $(810 \mathrm{~nm})$ [39].

2.1.2. Photochemical Stability. When excitated ICG is supposed to produce singlet oxygen, which is a strongly cytotoxic agent. Engel et al. have recently studied the stability of ICG when exposed to light and the production or the consequences of singlet oxygen production of ICG [7]. According to their observations the decomposition of ICG is due to singlet oxygen, but it seems that the singlet oxygen is immediately bound to the decomposition products of ICG itself. Therefore, it seems that ICG is not a very good source of singlet oxygen. This has two main consequences with respect to clinical applications: firstly, ICG can be used without much worry of phototoxicity due to singlet oxygen production; secondly, when ICG is used as a photodynamic or photothermal agent, its decomposition products may be the main cause of phototoxicity. The decomposition products thermally decompose further to several carbonyl compounds. However, according to a recent study by Tokuda et al., ICG seems to be somewhat phototoxic for the retina [40].

Engel et al. tested several solvents for light-induced decomposition of ICG. What is again interesting and encouraging for angiography applications is that ICG in blood plasma was found to decompose so that only a small amount of decomposition products were recorded when compared to ICG in water. They suggest that the singlet oxygen produced is quenched by some plasma proteins thus inhibiting ICG decomposition by singlet oxygen [7]. Very recently Sato et al. studied the effect of broadband light on ICG toxicity by filtering the long wavelengths focused on cultured Müller cells. According to their observations filtering prevents phototoxicity [41].

2.1.3. Protein Binding and Fluorescence Life-Time. The important property of fast binding to plasma proteins, especially lipoproteins, [42-44] makes repeated intraoperational applications of ICG possible. The binding to plasma proteins does not seem to alter protein structures, which is one sign of nontoxicity [45]. It seems that ICG actually binds to the lipids of lipoprotein complexes ( $\beta$-lipoprotein [43]), and that the bind results in more intense fluorescence than ICG bound to for example, free cholesterol [44]. Binding to 
TABLe 3: ICG cytotoxicity studies.

\begin{tabular}{lccc}
\hline Cell & Type & Ref. & Comment \\
\hline ARPE19 & In vitro & {$[54]$} & ICG 1 mg/mL; not toxic \\
RPE & In vitro & {$[55]$} & IfCG; no damage \\
Glial cell & Ditto & Ditto & ditto; some damage \\
RPE & Rabbit & {$[56]$} & ICG; no damage \\
RPE & Ditto & Ditto & IfCG; no damage \\
RPE & Gene expr. & {$[57]$} & Cell cycle arrest and apoptosis \\
Ditto & Ditto & Ditto & 0.25 mg/mL recommended \\
Müller & In vitro & {$[41]$} & Fluorescent lamp illumination: \\
Ditto & Ditto & Ditto & induces cytotoxicity \\
Intravenous & Rat & {$[58]$} & IRDye 800CW; no toxicity obs. \\
Intradermal & Ditto & Ditto & Ditto; ditto \\
Spin. root ax. & Rat & {$[59]$} & Neurotoxicity observed \\
RPE & Human cult. & {$[60]$} & Growth inhibition and damage \\
RPE & Human cult. & {$[61]$} & ICG interactions with RPE \\
\hline
\end{tabular}

blood proteins also shifts, slowly, taking several minutes, the absorption peak, at $780 \mathrm{~nm}$, towards longer wavelengths, to $805 \mathrm{~nm}$ [46]. The absorption peak maximum was observed at $810 \mathrm{~nm}$ in the epidermal cell cultures [39], and at 805$810 \mathrm{~nm}$ in the human skin in vivo $[47,48]$. The emission peak is also shifted similarly [14]. Not only the shape of the spectra is influenced by the chemical environment, but also the fluorescence life-time changes, a fact which can be used to probe the molecular environment of ICG and similar dyes [49].

2.2. Physiology and Pharmacokinetics. ICG does not have any known metabolites, and it is fast extracted by the liver into bile juice. The transport is done by a protein called glutathione S-transferase without [7] modification. Caloric restriction seems to significantly increase the plasma clearance rate at low doses $(0.5 \mathrm{mg} / \mathrm{kg})$ [50]. The protein spectra of different liver diseases also affect ICG protein binding in blood $[43,51]$. Reekers et al. provide a recent study of the plasma disappearance rate for ASA physical status I-II patients [52].

The typical dye concentrations used for in vivo retinal and choroidal angiography are in the range of $20-25 \mathrm{mg} / \mathrm{mL}$ of ICG applied by injection into a peripheral arm vein [46]. For studies of hepatic function an intravenous injection dose is calculated on the basis of $0.5 \mathrm{mg} / \mathrm{kg}$ of body weight. In cardiac output and blood volume monitoring the total dose of dye injected should be kept below $2 \mathrm{mg} / \mathrm{kg}$. No significant toxic effects have been observed in humans with the high dose of $5 \mathrm{mg} / \mathrm{kg}$ of body weight [53]. Table 3 gives a brief overview of toxicity studies done with ICG.

2.3. Penetration. ICG works in the so-called tissue optical window, that is, the NIR light used both in excitation and fluorescence penetrates tissue several millimeters or even further. This translucency helps to observe, for example, vascular structures that might be buried in clots or dura $[62,63]$. The penetration depth of light energy into skin and underlying tissues can be calculated on the basis of in vivo measurements of optical density $O D$ (accounting scattering and absorption) of the human skin and underlying tissues defined as $O D=\log _{10} I_{0} / I_{t}$, where $I_{0}$ is the measured back reflected intensity, and $I_{t}$ is that of the reference. Such measurements done for 12 healthy young subjects at 775 , 807 , and $827 \mathrm{~nm}$ gave the following dependencies for a $3 \mathrm{~mm}$ layer of subcutaneous fat: $O D_{775}=3.2 ; O D_{807}=2.4$; $O D_{827}=1.6[64]$. The low absorption and high scattering allow for providing smooth and intensive enough indepth irradiation of skin tissue due to the photon recirculation effect [65].

2.4. ICG Derivatives and ICG-Like Contrast Agents. While NIR fluorescence (NIRF) imaging has recognised potential, only ICG is a clinically approved NIRF dye. Perhaps in the future there will be a larger set of NIRF dyes. At least work on developing new NIRF dyes has been going on and has already introduced several potential NIRF dye candidates. Here we will only briefly review some recent development of ICG derivatives.

While ICG is rapidly bounded with lipoproteins in blood, it is natural to combine ICG with nanoparticles of lipoproteins [66]. Lipid nanoparticles and micelles have been doped with ICG [67-70]. Ogawa et al. have conjugated ICG with several antibodies in order to target ICG to cancer cells. However, ICG conjugated to protein usually markedly looses its fluorescence. Therefore, in order to have high fluorescence efficiency, the ICG-antibody complex should be dissociated so that ICG can be used as an in vivo molecular imaging probe [71]. S. Achilefu's group has recently conjugated ICG with folate-polyethylene glycol for tumor targeting [72]. Ebert et al. have compared the pharmacokinetics of ICG to its hydrophilic derivative called SIDAG with a mice model for breast cancer imaging [73].

Several encapsulations have been implemented with ICG [74-78]. Makino et al. have labeled lactosome with ICG. The labeled lactosome was found to be stable in blood circulation 
and gradually accumulated specifically at a mouse model liver tumor site [79]. Barth et al. have engineered calcium phosphosilicate composite nanoparticles embedding ICG for targeting human breast and pancreatic cancers [80].

Infracyanine green (IfCG) (Laboratoires SERB, Paris, France), also known as IFC green, is ICG without iodine. It is believed that IfCG is less cytotoxic in macular applications because $5 \%$ glucose solution instead of pure water is used as its solvent. According to [56], IfCG is less than ten times as retina cytotoxic as ICG. Infracyanine green was used in the study of macular pucker surgery [81]. The absorption and emission spectra of the commercial ICG and IfCG products in several solvents and concentrations are compared in [82].

\section{Instrumentation}

In this section, an overview of ICG imaging from the instrumentation engineering point of view will be given. Indocyanine green imaging belongs to the class of optical fluorescence imaging. Correspondingly, when used with an operational microscope it closely resembles fluorescence microscopy. Thus, the instrumentation needed is similar or even exactly the same as that for fluorescent imaging in general, or fluorescence microscopy in particular.

3.1. Fluorescence Imaging. As a rule, fluorescence microscopy is done so that both visible or excitation and fluorescence images are displayed together as one image. The fluorescence image alone may contain only a few details so that the visible image greatly helps to locate the fluorescing parts with the help of the landmarks seen in the visible image. Typically the fluorescence channel is shown, rendered, in colors like vivid green, having a striking colour contrast to the visible image of tissues. This kind of visualisation is especially important in intraoperational use, where the fluorescing parts, like blood veins, should be recognised easily and immediately. In order to be able to combine the two images, they should be aligned correctly. This is called image registration, and it is generally a computationally hard image processing operation $[83,84]$, while the rendering of the two images for display is a straightforward operation.

However, the image registration problem can be totally avoided by optical means by using an ordinary beamsplitter, which is a dichroic mirror splitting and filtering the beam into two parts: one for the visible camera and the other for the NIR camera. This means that both cameras see exactly the same field of view (FOV), and no registration is needed, provided that the cameras have identical optics and are located correctly with respect to each other. In addition to the beamsplitter, suitable exchangeable filters embedded in the optics or in a separate filter cube, which is the usual arrangement in fluorescence microscopy, are used in front of the cameras to block unwanted wavelengths from entering the sensors [85]. The filter is especially important for the NIR camera, so that the excitation light does not mix with the fluorescence signal because both are summed at the sensor and inseparable in the resulting electronic image. Visible range cameras usually already contain filters that block most of the NIR radiation that would otherwise be summed

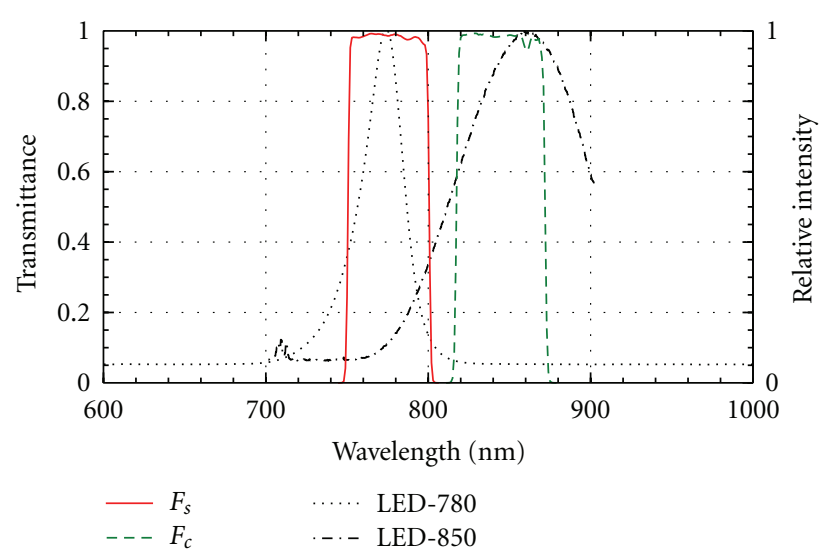

FIGURE 3: The transmission of the ICG filter pair $\left(F_{s}\right.$ : high-pass filter for source; $F_{c}$ : low-pass filter (barrier) for camera) and the emission spectrum of two NIR LEDs having the nominal peak wavelengths of 780 and $850 \mathrm{~nm}$ and full width at half maximum (FWHM) bandwidths correspondingly of 30 and $95 \mathrm{~nm}$.

to a varying extent to the different RGB channels of the visual image, thus distorting its perceived colours. The main difference between a traditional fluorescence microscope and an operational microscope doing ICG fluorescence imaging with a beam splitter is that the illumination, which is episcopic in the fluorescent microscope and thus done via the beamsplitter, is replaced by an ordinary colour camera, while the illumination can be done by an external light source [86]. The excitation light should not contain fluorescence wavelengths, they should originate only from the fluorescing ICG. Thus, a filter is needed to block longer wavelengths from the launched excitation light, when using a broad spectrum light source. Ideally the two filters should divide the spectrum into two nonoverlapping bands (Figure 3). This can be best done using interference filter pairs, which can be tailored for any wavelength range and which can have a very narrow transition band. Commercial interference filter pairs are also available for ICG fluorescence, separating the spectrum at about $800 \mathrm{~nm}$ (Chroma Technology, Brattleboro, VT, USA) (see Figure 3). When using a light source with a narrow spectrum, a laser, there is no need to use any excitation light filter. The use of a beamsplitter is a particularly simple and practical way of solving the otherwise challenging image registration problem and allows an illustrative blending of the colour image and the ICGA to be easily done online, which is often vital for critical intraoperational use (Table 4).

3.2. Example of an ICGA Device Design. In this section, we will, in principle, design a simple ICGA device. The principle of fluorescence imaging is given in Figure 4. In order to see the fluorescence, which has only a fraction of the intensity of the excitation light, the latter should not contain any fluorescence wavelengths. If a broadband light source, like a halogen lamp, is used, there should be a filter to cut the longer wavelengths $\left(F_{S}\right)$. In the case of using lasers, which are monochromatic, no filter is usually needed. On the camera side complementary filtering is always needed, that is, 
TABLE 4: ICGI instruments. KAIST: Korea Advanced Institute of Science and Technology.

\begin{tabular}{lccc}
\hline Manufacturer & Device & Ref. & Comment \\
\hline Carl Zeiss & Pentero IR-800 & {$[87]$} & Surgical micr. \\
Carl Zeiss & Pentero? & {$[88]$} & Neurosurg. micr. \\
Cri, Inc. & Maestro & {$[71]$} & Small animal \\
Eastman Kodak & Imaging Station FX & {$[89]$} & Small animal \\
Eastman Kodak & Ditto 4000 MM & {$[90]$} & Small animal \\
Florida Int. U. & Prototype FIU $U_{1}$ & {$[91]$} & Breast imager \\
Hamamatsu & PDE & {$[92]$} & \\
Hamamatsu & Prototype $\left(H_{1}\right)$ & {$[93]$} & Laparoscopic \\
KAIST & Prototype AA $A_{1}$ & {$[94]$} & Small animal \\
Mizuho Ikakogyo & HyperEye & {$[95]$} & surgery \\
Novadaq Tech. & SPY & {$[96]$} & \\
Osaka Med. Coll. & Prototype OMC & {$[97]$} & Neurosurg. micr. \\
Pulsion & IC-View & {$[98]$} & \\
Topcon & TRC-50IX & {$[99]$} & Ophthalmoscope \\
U. Clinic Munich & Prototype $U C M_{1}$ & {$[67]$} & Endoscope \\
U. Kent & Prototype $U K_{1}$ & {$[100]$} & OCT ophthalm. \\
Vieworks Corp. & VasView & {$[101]$} & Human leg im. \\
Wetzlar & Leica OH3 FL800 & {$[102]$} & Surgical micr. \\
\hline
\end{tabular}

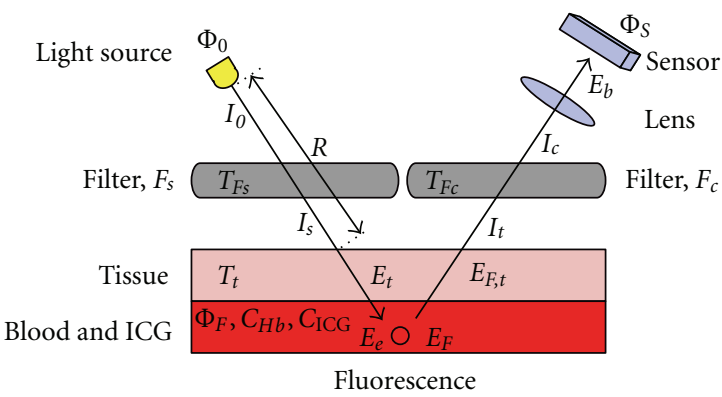

FIgURE 4: The principle of fluorescence imaging. The radiation from the light source is filtered by a high-pass filter, $F_{s}$, to remove the fluorescent wavelengths. The blood and ICG suspension under a tissue absorbs the excitation wavelengths and emits in fluorescent band. The emitted light is received by the sensor through a low-pass filter, $F_{c}$, to remove the excitation light reflected from the source.

the excitation wavelengths, and possibly shorter wavelengths should be cut off $\left(F_{c}\right)$. As can be seen in Table 5, there is some freedom when selecting the exact filter wavelengths. In addition to the filter wavelengths we should also look at the wavelength dependence of the light sources, filters, and cameras. In an ideal case no excitation light is recorded by the camera while as much as possible of the fluorescence is recorded. This is obviously a two-goal optimisation problem: minimise excitation light leakage while maximising recorded fluorescence light. What makes this a nontrivial technical problem is the shape of the spectra of each component needed and the other properties of the components affecting recording. For example, the quantum efficiency of the silicon semiconductor-based image sensors in NIR band typically
TABLE 5: ICGI instrument properties. ${ }^{*}$ : Hitachi, $\lambda_{e}[\mathrm{~nm}]$ emission wavelength $(\mathrm{min}), \lambda_{c}[\mathrm{~nm}]$ camera wavelength $(\mathrm{min})$, and $\mathrm{CCCD}$ cooled CCD.

\begin{tabular}{lccccc}
\hline Device & Light & $\lambda_{e}$ & Camera & $\lambda_{c}$ & Ref. \\
\hline$A A_{1}$ & LED & 740 & cCCD & 820 & {$[94]$} \\
FX & Halogen & 755 & cCCD & 830 & {$[89]$} \\
$H_{1}$ & Xenon & $?$ & CCD & 810 & {$[93]$} \\
IC-View & LED? & 780 & CCD? & 835 & {$[98]$} \\
Maestro & $?$ & 710 & $?$ & 800 & {$[71]$} \\
OMC & Halogen & 760 & KP-160* & 820 & {$[97]$} \\
Pentero? & Laser & 780 & $?$ & 835 & {$[88]$} \\
PDE & LED & 760 & CCD & 820 & {$[103]$} \\
VasView & LED & 760 & CCD & 830 & {$[101]$} \\
UK $K_{1}$ & SLD & 793 & $?$ & 807 & {$[100]$} \\
\hline
\end{tabular}

TABLE 6: Light source properties.

\begin{tabular}{|c|c|c|c|}
\hline Property & Halogen & LED & Diode laser \\
\hline Wavelengths & Visual-NIR & Rather narrow & Monochromatic \\
\hline Price & Cheap & Cheap & $\begin{array}{l}\text { Relatively } \\
\text { expensive }\end{array}$ \\
\hline Maintenance & Some & Not much & Some \\
\hline Power & High & Rather high & High (pulses) \\
\hline Pulses & Mechanically & Electronically & Electronically \\
\hline Speed & Slow & Quite fast & Slow-very fast \\
\hline Stability & Poor & Good & Good-very good \\
\hline Special & Visual imaging & Small size & $\begin{array}{l}\text { Extreme } \\
\text { performance }\end{array}$ \\
\hline Benefits & Cheap & $\begin{array}{l}\text { Easy to } \\
\text { control }\end{array}$ & No filtering needed \\
\hline \multirow[t]{2}{*}{ Drawbacks } & $\begin{array}{l}\text { High power } \\
\text { loss }\end{array}$ & Filter needed & Speckle pattern \\
\hline & Filter needed & New tech. & White light needed \\
\hline
\end{tabular}

strongly decreases when the wavelength increases. The quantum efficiency means the fraction of photons striking the sensor that are actually recorded. For silicon sensors, it is in the visible wavelengths typically around $70 \%$, while for NIR wavelengths around 700-900 nm it may range from 50\% down to $10 \%$ or even less (Figure 5).

3.2.1. Light Sources. Table 6 describes the basic properties of light sources available for fluorescence imaging. As we have seen in Table 5, all the basic light source types have been used in some of the existing ICGA implementations. Most frequently LEDs and halogen lamps have been used for illumination. In some experiments also lasers, mainly semiconductor diode lasers, resembling LEDs, have been used. In this example, we will look closer at LEDs. LED light is not totally monochromatic but contains wavelengths typically having a bell-shaped spectrum (Figure 3), which should not overlap too much with the camera filter spectrum $\left(F_{c}\right)$ (Figure 3). 
TABLE 7: Some commercial NIR camera sensors.

\begin{tabular}{lcccc}
\hline Sensor & Technology & Resolution & Application & Manufacturer \\
\hline MT9P031 & CMOS & 5 Mpix & Consumer & Aptina \\
MT9V032 & CMOS & 0.36 Mpix & Surveillance & Aptina \\
KAI-11002 & CCD & $10 \mathrm{Mpix}$ & Consumer & Kodak \\
Neo & sCMOS & $5.5 \mathrm{Mpix}$ & Scientific & Andor \\
iXon3 & EM-CCD & $1 \mathrm{Mpix}$ & Scientific & Andor \\
FL-280 & sCMOS & $2.8 \mathrm{Mpix}$ & Medical & Hamamatsu \\
ER-150 & CCD & $1.3 \mathrm{Mpix}$ & Medical & Hamamatsu \\
\hline
\end{tabular}

TABLE 8: The most important pixel parameters of the above NIR camera sensors.

\begin{tabular}{lccccc}
\hline Sensor & $\begin{array}{c}\text { ADC } \\
\text { resolution } \\
\text { (bit) }\end{array}$ & $\begin{array}{c}\text { Read } \\
\text { noise } \\
(e)\end{array}$ & $\begin{array}{c}\text { Dark } \\
\text { current } \\
(e / \text { pix/s })\end{array}$ & $\begin{array}{c}\text { Pixel } \\
\text { size } \\
\mu \mathrm{m}^{2}\end{array}$ & $\begin{array}{c}\text { Well } \\
\text { depth } \\
\cdot 1000 e\end{array}$ \\
\hline MT9P031 & 12 & 2.6 & 25 & 4.8 & 8.5 \\
MT9V032 & 10 & - & - & 36 & - \\
KAI-11002 & 16 & 17 & - & 9 & 60 \\
Neo & 16 & 1 & 10 & 6.5 & 25 \\
iXon3 & - & $<1$ & - & 13 & 80 \\
FL280 & 12 & 3 & - & 13 & 18 \\
ER-150 & 12 & 10 & - & 41 & 15 \\
\hline
\end{tabular}

If a visual image is recorded or observed, we naturally need a white light source. Note, that most microscope lights filter out NIR wavelengths at least partly.

Figure 3 shows the spectrum of an LED having a nominal (peak) wavelength of $780 \mathrm{~nm}$ (LED 780-66-60, Roithner Lasertechnik GmbH, Vienna, Austria). The measurement was done with an HR4000 Spectrophotometer (Ocean Optics, Dunedin, FL, USA). As can be seen, the wavelength range is over one hundred nanometers with $30 \mathrm{~nm}$ bandwidth (FWHM).

3.2.2. Cameras. Every CCD or CMOS camera is, in principle, able to record NIR. However, most cameras are prevented from doing so by a filter that cuts NIR wavelengths, otherwise the superimposed NIR image would badly interfere with the visual image. The most important parameters of the camera sensors are resolution, signal to noise ratio (SNR), and quantum efficiency. The parameters affecting the SNR are the resolution of the ADC converter, read noise, dark current, and quantum well depth of the sensor. These parameters for some selected sensors are listed in Tables 7 and 8: MT9P031 and MT9V032 (Aptina Imaging, San Jose, CA, USA) are typical complementary metal oxide semiconductor (CMOS) sensors, except that the NIR response of the latter is enhanced. The machine vision camera, Elphel NC353L (Elphel Inc. West Valley City, Utah, USA) includes the MT9P031 sensor. The KAI-11002 (Kodak, New York, USA) is a typical Charge Coupled Device (CCD) sensor. iXon3 and Neo sensors (Andor Technology plc, Belfast, North Ireland) are meant for scientific imaging, where high sensitivity is

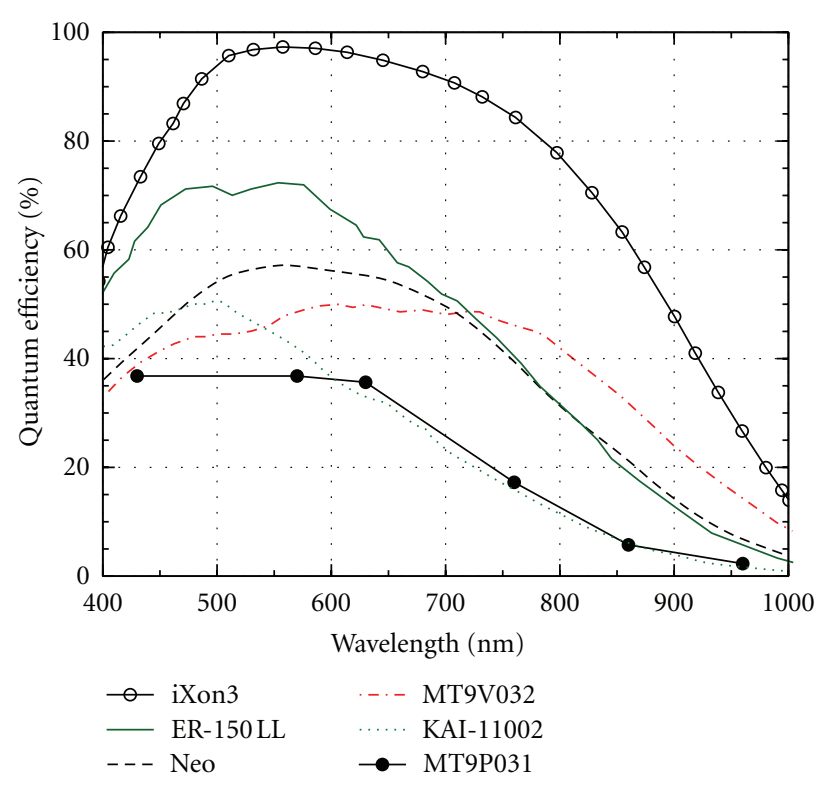

FIGURE 5: The quantum efficiencies of different sensor technologies in VIS-NIR range. iXon3 is an electron multiplier CCD, ER-150 LL is Hamamatsu biomedical CCD sensor, Neo is a scientific CMOS sensor, MT9V032 is a CMOS sensor for surveillance, KAI-11002 is a standard CCD sensor, and MT9P031 is a standard consumer CMOS sensor.

needed. The iXon3 is based on Electron Multiplier CCD (EM-CCD) technology, whereas Neo is based on the scientific CMOS (sCMOS) sensor. The FL-280 and ER-150 are corresponding sCMOS and CCD sensors from Hamamatsu (Hamamatsu Photonics K.K, Shizuoka, Japan).

While practically all silicon-based cameras are somewhat sensitive to near-infrared, when they do not have a filter to block NIR wavelengths, unfortunately the quantum efficiency tends to decrease quite rapidly by increasing wavelength (Figure 5). This decrease of quantum efficiency is an essential issue when designing ICG imaging because the fluorescence peak is quite broad and extends far beyond $800 \mathrm{~nm}$, where the efficiency is quite low. Therefore, the nominal wavelengths of the light source and filters should be as short as possible, which is in contradiction with the good separation of excitation and fluorescence and the fact that the absorption maximum of ICG should be close to the nominal wavelength of the excitation light source. The quantum efficiency of ICG is quite low, about $0.3 \%$ in water and $1.2 \%$ in blood [104]. This constrains camera sensitivity especially in video applications. Cooled CCDs are often used to increase the signal to noise ratio (cf. Table 5). Sometimes image intensifiers (night vision) are used to increase sensitivity for video recording and to allow low doses of ICG (microdosing) [21, 27, 105, 106].

3.2.3. Filter Design. For the LED source of Figure 3 we need a filter that effectively blocks all wavelengths longer than $800 \mathrm{~nm}$. It seems that the most suitable off-the-shelf filter can be found from Chroma (Chroma Technology, Brattleboro, VT, USA), specially tuned to ICG fluorescence. The filter 
TABLE 9: An example of the light attenuation in an ICG imaging system.

\begin{tabular}{clcc}
\hline Row & Component & Attenuation & Remaining intensity \\
\hline 1 & LED 780-66-60 & 0.2 & $I_{0}=1 \mathrm{~W} / \mathrm{sr}$ \\
2 & Fs, ET775_50x & 0.8 & $I_{s}=0.8 \mathrm{~W} / \mathrm{sr}$ \\
3 & Tissue irradiance & & $E_{t}=80 \mathrm{~W} / \mathrm{m}^{2}$ \\
4 & Tissue, 1 mm & 0.45 & $E_{e}=36 \mathrm{~W} / \mathrm{m}^{2}$ \\
5 & Fluorescence & 0.0027 & $E_{f}=0.10 \mathrm{~W} / \mathrm{m}^{2}$ \\
6 & Tissue, 1 mm & 0.45 & $45 \mathrm{~mW} / \mathrm{m}^{2}$ \\
7 & Lambertian S. & 0.32 & $I_{t}=14 \mathrm{~mW} / \mathrm{sr}$ \\
8 & Fc, ET845_55 m & 0.3 & $I_{c}=4.3 \mathrm{~mW} / \mathrm{sr}$ \\
9 & Irradiance, f/1.1 & 0.82 & $E_{b}=3.5 \mathrm{~mW} / \mathrm{m}^{2}$ \\
\hline & Response of the Hamamatsu ER-150 low light \\
\hline 10 & Power per pixel & & $P_{p}=0.15 \mathrm{pW}$ \\
11 & Photons per pixel & & $N_{p}=6.1 \cdot 10^{5} \mathrm{photons} / \mathrm{s}$ \\
12 & Detected photons & 0.25 & $N_{e}=1.5 \cdot 10^{5} \mathrm{electrons} / \mathrm{s}$ \\
13 & Optimal exposure & & $t=98 \mathrm{~ms}$ \\
14 & Signal to noise & & $\mathrm{SNR}=63 \mathrm{~dB}$ \\
\hline
\end{tabular}

HQ845/55 m, which is of the interference type, has quite a sharp pass band between 820 and $870 \mathrm{~nm}$, and when comparing the spectra of the LED (LED: 780, 66, and 60) we are using and this filter we can see that their spectra somewhat overlap (Figure 3).

A rigorous approach to ICGA system design would include numerical analysis of the spectra of the light source, filters, and the camera in order to find the optimal nominal wavelength of the components. However, in this study we have simply resorted to those components that were easily available and which seemed to fit with each other well enough.

Photometric formulas can be still used in developing practical rules of thumb to estimate the effect of different components of the ICG imaging system as follows.

The radiant flux of illumination source (cf. Figure 4), $\Phi_{0}$ [W], distributed over a solid emission angle $\Omega_{0}[\mathrm{sr}]$, forms the radiant intensity of $I_{0}=\Phi_{0} / \Omega_{0}$ [W/sr] (Table 9; row 1). $I_{0}$ travels through the excitation filter, $F_{s}$, the transmittance of which is $T_{F_{s}}$ [unitless], attenuating to $I_{s}=I_{0} T_{F_{s}}$, (Table 9; row 2) and hits the tissue surface. The irradiance of the tissue, $E_{t}\left[\mathrm{~W} / \mathrm{m}^{2}\right]$, depends on the distance, $R[\mathrm{~m}]$, and angle of the incidence, $\theta$, as follows: $E_{t}=I_{s} \Omega_{0} \cos (\theta) / R^{2}$. The tissue attenuates the incident irradiance by the factor of $T_{t}$. For $1 \mathrm{~mm}$ of aorta tissue, the $T_{t} \approx 0.45[1 / \mathrm{cm}]$ [107] (Table 9; row 4).

Part of the incident irradiance is absorbed by blood and ICG and part of it will be diffusely reflected due to the scattering of the red blood cells (RBCs). The intensities of the excitation and fluorescence fields can be calculated using diffusion theory [108-110]. The scattering coefficient of red blood cells and absorption coefficients of hemoglobin, needed in the diffusion model, are listed in [111, 112]. Correspondingly, the absorption properties of ICG are listed in [113]. According to diffusion theory, the diffuse reflectance, excluding the fluorescence, is $R_{d} \approx 0.15$. When the fluorescence is included, the total reflected and emitted intensity $R_{t}$ is slightly higher. The intensity of the fluorescence emission is $E_{f}=\left(R_{t}-R_{d}\right) E_{e} \approx 0.0027 E_{e}$. Increasing the ICG concentration increases $E_{e}$ quite fast in low doses, for example, when $C_{\text {ICG }}=6.5 \mu \mathrm{M} / \mathrm{L}(0.31 \mathrm{mg} / \mathrm{kg})$. The intensity of fluorescence is nonlinear. It approximately doubles when the ICG concentration is increased ten fold, that is, $C_{\mathrm{ICG}}=$ $65 \mu \mathrm{M} / \mathrm{L}(3.1 \mathrm{mg} / \mathrm{kg})$.

The fluorescent light proceeds through the layer of tissue, which again attenuates the irradiance by the factor of $T_{t}$. The remaining irradiance is scattered from the surface of the skin. If a Lambertian surface is assumed, the radiant intensity emitted from the tissue, $I_{t}$, can be obtained from the irradiance of the skin, $E_{F, t}$, as follows: $I_{t}=E_{F, t} / \pi$ (Table 9; row 7).

Before hitting the sensor, the radiant intensity, $I_{t}$, is attenuated by the emission filter, $F_{c}$. Approximately $T_{F_{c}}=30 \%$ of the energy of the ICG fluorescent spectrum goes through the filter. Therefore, the intensity of light $I_{c}$ entering the camera optics is $I_{c}=I_{t} T_{F_{c}}$. (Table 9; row 8). The performance of the optics is often expressed as the so called $f$-number. The radiance in the image plane, $E_{b}$, is obtained from the radiant intensity using the $f$-number of the optics, as follows: $E_{b}=$ $I_{t} / f^{2}$ (Table 9; row 9).

As we have seen, only a small fraction of the initial light intensity induces fluorescence which finally will reach the image plane. To compensate the low light intensity, the exposure time, $t[s]$, must be relatively long, which increases the signal level, $S=\Phi_{s} E_{b} t$, where, $\Phi_{s}$, is the quantum efficiency of the sensor. Long exposure time also increases the level of thermal noise, $N_{\text {th }}$ [e/pixel], due to dark current, $i_{d}[\mathrm{e} / \mathrm{pixel} / \mathrm{s}]$. The total thermal noise within the exposure interval, $N_{\mathrm{th}}=i_{d} t$, dominates the total noise, $N_{r}$ [e/pixel], when $t>N_{r} / t$. Above this limit, increasing the exposure time increases the total noise level, $N_{\text {tot }}=N_{r}+N_{\text {th }}$, approximately at the same rate as the signal level, and thus the signal to noise ratio, $\mathrm{SNR}=S / N_{\text {tot }}$, is not significantly improved any more. Therefore, the light entering the image plane should have sufficient intensity to keep the exposure time short enough.

The optimal exposure time $t_{o}$ can be determined, if the physical pixel area, $A_{p}$, and the maximum number of electrons the pixel can hold, the quantum well depth, $D_{\mathrm{QW}}$, are known. The number of photons hitting the pixel is $E_{b} A_{p} / E_{p}$, where $E_{p}=c h / \lambda$ is the energy of the photon, where $c$ is the speed of light, $h$ is Plank's constant, and $\lambda$ is the wavelength of the photon. Therefore, the time which is needed to fill the quantum well is

$$
t_{o}=\frac{D_{\mathrm{QW}} E_{p}}{\Phi_{s} E_{b} A_{p}} .
$$

As an example, the calculation of the observed fluorescent intensity and the performance of the Hamamatsu ER-150 sensor is estimated in Table 9 (rows 10-14). Many parameters used in the calculation are only estimations, especially the tissue properties above blood layer, the haemoglobin concentration in blood, the concentration of the ICG and the quantum efficiency of ICG. Furthermore, the fact that the emission spectrum of ICG may depend on 
TABLE 10: The loss factors and corresponding attenuations $[\mathrm{dB}]$ of the top five loss factors in ICGA imaging.

\begin{tabular}{lcc}
\hline Loss factor & Loss & $\mathrm{dB}$ \\
\hline The fluorescence of ICG in blood & 0.0027 & -25.6 \\
Losses in the tissue above the blood vessel & 0.2 & -6.9 \\
Quantum efficiency of sensor $\Phi_{s}$ & 0.25 & -6.0 \\
Transmittance of the emission filter & 0.3 & -5.2 \\
Diffusion losses in the lambertian surface & 0.32 & -5.0 \\
\hline Subtotal & $13.4 \cdot 10^{-6}$ & -48.7 \\
\hline Other factors together & 0.62 & -1.7 \\
\hline Total & $8.8 \cdot 10^{-6}$ & -50.5 \\
\hline
\end{tabular}

the spectrum of the illumination is neglected. Therefore, the absolute values given in the above calculations are not accurate. However, they provide insight into the losses in the imaging system. Eventually only about 35 ppm (parts per million) of the original radiation intensity remains in the image plane, and only about 25 percent of it will be detected. The summary of top five loss factors causing $-48.7 \mathrm{~dB}$ attenuation of the total $-50.5 \mathrm{~dB}$ attenuation in the example system is shown in Table 10. Remember that one full stop used in camera lenses is equivalent to $3 \mathrm{~dB}$. Thus, the total attenuation corresponds to about 17 full stops, which means that we need to use large aperture, long exposure time, and strong illumination in order to get high quality, that is, high SNR ICG fluorescence images.

3.2.4. Test and Background Light. As NIR light is not visible to the human eye and fresh ICG-water solution is not always at hand, it is practical to have a test light to see if the camera system is working on the ICG fluorescence wavelengths. We have used an LED SFH485-P (Osram/Siemens, Berlin, Germany), having peak emission at $880 \mathrm{~nm}$, as a test light to see if the camera is tuned to wavelengths ranging from about $800 \mathrm{~nm}$ to $900 \mathrm{~nm}$. We have also constructed a simple light control for using this LED as a background light for ICG fluorescence imaging. The test light can also be used as tunable backlight, when we want to see landmarks not fluorescing themselves.

3.2.5. Optics. Our example system was based on an old operational microscope originally not at all designed for NIR imaging (Wild, Figure 6). It has two oculars and a $C$ mount for a camera for both eyes. This gives us an opportunity not only to record ICGA videos but also ICGA stereo videos. It has been shown that stereo videos are beneficial in the training of surgery students [114].

Excluding the microscope, the cost of our prototype components including two interference filters, two cameras, an LED light, and a PC with some software is about 3000 euros. Figure 1 shows a typical image taken by our prototype system. As can be seen the quality of the image is quite good, especially when we remember that the optics used (the microscope) is not designed for NIR imaging. The use of special NIR optics would considerably increase the cost of

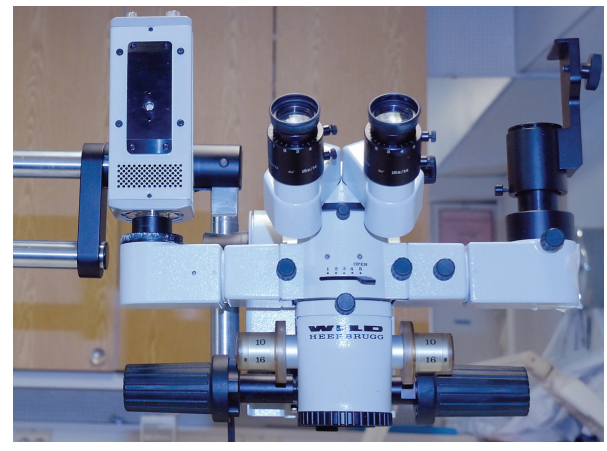

Figure 6: An old operational microscope used in our prototype ICG stereo video angiography system experiment. Hamamatsu NIR camera on the left camera arm.

the system while probably only somewhat increasing the image quality.

3.2.6. ICGA Test. After technical laboratory tests, our device was tested by recording ICGA of rat heart (Figure 4). Neither the use of ICG dye nor the modest optics not originally designed for NIR imaging does too much restrict resolution, when compared to imaging in visible wavelengths: arteries, the caliper of which are only a fraction of millimeter, can be clearly seen.

Using two cameras, stereo images and stereo video can be taken. The cameras can be attached to an operational microscope (Figure 6) or simply attached together when microscopy is not used. This gives literally new vision for ICGA, especially for educational and training purposes when complex scenes can be seen stereoscopically.

\section{Surgical Applications}

Established medical applications of ICG are retinal angiography, liver clearance test, and cardiac output monitoring. ICG is fast removed from circulation by the liver into bile juice, which is applied in liver condition monitoring. It also gives the option to inject ICG several times during an operation if needed. Recent interest in ICG is based on new applications in surgery and especially in angiography related to intraoperative monitoring of blood circulation in vital organs, where intraoperative angiography is also economically motivated [115].

4.1. Intraoperational Angiography. As compared to other angiography methods (X-ray, CT, MRI, and PET), ICGA can be easily and economically used intraoperationally, when blood vessels are exposed allowing direct visual observation, for example, in neurosurgery, bypass coronary surgery, flap operations in reconstructive surgery, wound and trauma surgery, and laparoscopic surgery, where it is vital to check that blood circulation is recovered properly.

The imaging protocol is simple, and devices are relatively cheap. ICG is given as an injection (bolus) into systemic blood circulation and imaging is done during a period of few 
minutes after injection. Normally a new bolus can be given after about 15 minutes.

4.2. Neurosurgery. Neurosurgery is ideal for ICGA because operations are already done under a microscope (and camera), and because the blood veins located on the brain surface are mainly exposed and thus can be seen more or less directly by visual means. Milestones in neurosurgery include

(i) 2001: experiment with surgical microscope (OPMI) in neurosurgery [97];

(ii) 2002: FDA approval for cerebral angiography research [102];

(iii) 2003: ICGA was introduced for clinical neurosurgery [88];

(iv) 2005: ICGA done with surgical microscope [116];

(v) Leica 2006: FDA approval of ICGA surgical microscope;

(vi) Zeiss 2007: commercial surgical microscope with ICGA;

(vii) Zeiss 2009: ICGA dynamics display software.

Earlier, ICG has been used in neurology, for example, for measurement of cerebral blood flow in newborn infants [117].

Neurosurgical vascular operations are usually performed to exclude vascular malformations from the circulation or to provide revascularisation in case of compromised cerebral perfusion. Typical vascular anomalies to be treated surgically are cerebral aneurysms and intracranial or intraspinal arteriovenous malformations (AVMs) and fistulas. It is of utmost importance to be able to verify that the malformation in question has been completely obliterated and removed from the circulation, and just as critical is to ensure that physiological blood flow in associated and adjacent vessels remains uncompromised at the end of the procedure. In revascularisation, that is, bypass procedures, the patency of the vascular microanastomosis is likewise paramount to successful procedures. Incomplete obliteration of a ruptureprone aneurysm or AVM may result in a hemorrhage, and occlusion of a parent vessel or an anastomosis in an ischemic stroke; both of which may have catastrophic consequences for the patient. Postoperative angiography is useful in assessing the residual filling of the treated lesion, but in case of inadvertent vessel occlusion the result of postoperative imaging comes too late, and the ischemic brain or medullary lesion has already irreversibly occurred. Although it is possible to use intraoperative digital subtraction angiography (DSA) in the operating room, the setup takes a relatively long time, and thus DSA cannot be used routinely in every operation. Moreover, DSA is associated with a complication rate of up to $3 \%$, and its resolution is insufficient to demonstrate the occlusion of small $(<1 \mathrm{~mm})$ perforating arteries, which, despite their small caliber, may supply blood flow to critical neural structures in, for example, basal ganglia and the brain stem. In neurosurgery all complex operations are performed under high magnification of a surgical microscope, which provides an excellent hardware platform for implementing new optical solutions and to mount various external devices, such as video cameras.

ICG angiography was introduced to neurosurgery in 2003 [88] and has become a routine method for intraoperative evaluation of intracranial blood circulation. It has been used at the Department of Neurosurgery at Helsinki University Central Hospital since 2005 in approximately 300 operations every year. It provides real-time information about the patency of vessels of all sizes seen in the field of the surgical microscope. Its usefulness in intracranial aneurysm surgery has been recently assessed in several large patient series, in altogether 620 aneurysms [116, 118-121]. The uniform conclusion of all the reports was that the correlation between ICG angiography and postoperative angiography has been $90-95 \%$, in terms of aneurysm remnants and vessel branch stenoses or occlusions. In addition, ICG angiography has the added advantage of demonstrating small perforating artery occlusion intraoperatively, enabling the immediate correction of aneurysm clip placement [118]. However, ICG angiography may be inadequate in cases of giant, complex, or deep-sited aneurysms [119]. Atherosclerotic calcifications also limit its reliability in demonstrating, for example, aneurysm neck remnants.

During microneurosurgical treatment of brain or spinal arteriovenous malformations and dural arteriovenous fistulas, the dynamic visualisation of different phases of the blood flow by ICG angiography is helpful in the identification and differentiation of feeding arteries, arterialised draining veins, and normal veins, as well as the fistulous sites, during intraoperative orientation within the surgical field [87, 122124]. It should be noted, however, that especially brain AVMs often have complex 3D anatomy, and ICG angiography is only able to visualise vessels on the surface of the illuminated surgical field. Likewise, ICG angiography cannot, at present, be considered reliable in assessing possible residual AVMs, which still requires DSA either intra- or post-operatively.

ICG angiography has also been evaluated and found reliable in assessing the patency of microanastomoses in neurosurgical extracranial-intracranial revascularisation bypass operations [125]. It was also helpful in identifying the target recipient artery of sufficient diameter $(>1 \mathrm{~mm})$ in extracranial-intracranial bypass procedures performed via very small $(3 \mathrm{~cm})$ craniotomies [126]. ICG-VA has also been demonstrated to be useful in evaluating the patency of extracranial vertebral artery after surgical transposition and in localizing vertebral artery within its periosteal sheath during surgery of cervical neurinomas [127]. Very recently, Haga et al. have used ICG-VA for assessment of carotid endarterectomy [128]. Commercially available semiquantitative dynamic ICG fluorescence analysis system has also been recently suggested to be able to demonstrate impaired regional perfusion in patients with cerebral ischemia [129].

The usefulness of ICG angiography in microneurosurgical vascular operations is increasingly acknowledged, as more applications are developed, and more experience is gained. However, there is still room for technical developments, for example, in form of rapid and reliable flow dynamics analyses and easily processable and repeatable video playback 
loops, since rapid ICG reinjections generally suffer from lower contrast due to residual ICG inside the vessels.

4.3. Coronary Surgery. In principle, coronary arteries are also ideal for ICGA because they are located, like brain arteries, on the organ they supply blood to. The major milestones of ICG in coronary bypass surgery include

(i) 2002: a pig model of coronary bypass angiography with ICG [130];

(ii) 2004-2005: comparison of ICGA and ultrasound flow metering at University of Toronto;

(iii) 2005: FDA approval for ICGA device SPY for coronary angiography;

(iv) 2005-2009: GRIIP clinical trial (phase III) at Sunnybrook Health Sciences Centre.

Coronary artery bypass grafting (CABG) is the most frequent cardiac operation with annual rates of 400,000 procedures in the United States and 76,000 in Germany. During these operations verification of graft patency should be a key aspect, as immediate intraoperative graft failure occurs in up to $4 \%$ of grafts ( $8 \%$ of patients) [131]. At patient discharge the graft occlusion rate is $5-20 \%$ and up to $30 \%$ at one year after the operation [132]. Intuitively, eliminating intraoperative graft failure and technical failure should reduce cardiac mortality and morbidity in the short term and improve clinical outcome in the long term. Although conventional angiography remains the gold standard technique for assessing graft patency, it is rarely available in the operating room and consequently several other less invasive approaches have been advocated. The most commonly used intraoperative method is transit-time flowmetry (TTFM), which measures the mean flow of the bypass graft and calculates a pulsatility index of the flow pattern. TTFM is reliable and sensitive in detecting graft failure, but in several patients it might lead to unnecessary graft revision [132].

Near-infrared imaging (NIR) based on the intravascular ICG dosing has emerged as a novel method for graft patency assessment. Two main systems have been introduced.

Firstly, an indirect method in which the myocardial tissue perfusion is assessed by imaging an area of interest around a coronary vessel. In this imaging method, peak fluorescence intensity and temporal slope of fluorescence intensity in the tissue are measured [130]. This imaging method has been shown to agree with the result of the fluorescent microsphere imaging, which is the golden standard [133].

Secondly, a direct imaging of the grafts by visualising the graft lumen by ICG angiography. In an early study by Rubens et al., 20 patients were studied by intraoperative ICG angiography, and one patient (5\%) was identified as needing a graft revision [134]. Taggart et al. investigated 213 grafts with a revision rate of 4 grafts (1.9\%) acknowledged by ICG angiography [135]. Reuthebuch and colleagues published a graft revision rate of $4(3.7 \%)$ out of 107 patients [136]. Balacumaraswami et al. assessed the intraoperative graft patency of 533 conduits in 200 patients. Fluorescence imaging confirmed technical failure in $8(1.5 \%)$ conduits in
8 (4\%) patients, necessitating graft revision [137]. Takahashi et al. reported a study of intraoperative ICG angiography of 290 grafts, in which four grafts $(1.9 \%)$ were visualised to need a graft revision [138]. In a paper by Desai et al., a total of 348 coronary bypass grafts were studied by ICG angiograms. In $4.2 \%$ of patients information from the ICG imaging led to graft revisions that would have otherwise gone unrecognised $[139,140]$.

Intraoperative graft occlusion in CABG is a consistent finding affecting up to $5 \%$ of grafts. This probably causes difficulties in both the short and the long term. Detection of technical problems in the most vital graft, the internal thoracic artery is of utmost importance. Among the available techniques for assessing graft patency, intraoperative ICG angiography seems to provide a sensitive method compared to the mostly used method of TTFM. In a recently published randomised trial, 156 patients were randomised to go through ICG angiography or TTFM during CABG to assess graft function intraoperatively. One year after the operation, 43 out of 312 grafts were occluded $(13,8 \%)$, with no difference between the groups. Thus, ICG angiography seems to provide a novel technique in addition to the more acknowledged range of methods of intraoperative quality confirmation in coronary surgery [96].

4.4. Vascular Surgery. In vascular surgery, ICG fluorescence imaging has been studied in intraoperative assessment of graft patency, diagnostics of peripheral arterial occlusive disease and Raynaud phenomenon (RP) as well as in predicting wound healing after major amputation and to evaluate splanchnic circulation. Also, the usefulness of ICG angiography in evaluating angiogenesis in small animal models and in detecting the vulnerability of atherosclerotic plaque has been tested. In one study ICG imaging was used in the treatment of varicose veins with sclerotherapy.

In a preliminary report by Unno et al., 9 patients were recruited in an intraoperative angiography performed with PDE. At the end of the operation before wound closure, ICG was injected in a central intravenous line. ICG dye reached the leg artery about 30 seconds after the injection. In 8 out of 9 cases, ICG angiography showed good fluorescent signals as the ICG passed through the graft. In one case no fluorescent signal was detected and during revision a distal thrombosis was detected and repaired [141].

Kang et al. have proposed a perfusion rate model based on ICG dynamics, which they later apply to human patients to diagnose peripheral arterial occlusive disease (PAOD) with VasView [90]. PAOD patients and control subjects with normal vasculature were evaluated for lower extremity tissue perfusion using ICG perfusion imaging. The perfusion rates of the lower extremities with severe PAOD were significantly lower than those of normal controls. Even in cases of mild PAOD, the perfusion rates were lower compared to the control, while the conventional methods failed to detect mild functional impairment. These results collectively indicated that ICG perfusion imaging is an effective tool for diagnosis of PAOD, when compared to the golden standard of anklebrachial blood pressure ratio [101, 142]. 
In a recent study, Kang et al. tested the use of combined analysis of multiple parameters, especially onset time and modified $T_{\max }$, which means the time from onset of ICG fluorescence to $T_{\max }$, to diagnose Raynaud phenomenon (RP). To validate the method, they performed a conventional thermographic analysis combined with cold challenge and rewarming along with ICG dynamic imaging and segmental analysis. A case-control analysis demonstrated that the segmental pattern of ICG dynamics in both hands was significantly different between normal and RP cases, suggesting the possibility of clinical application of this method for the reliable diagnosis of Raynaud phenomenon [143].

In patients with no possibility of revascularization, about half sustain amputation within one year. To maintain best possible mobility, amputation should be done as distally as possible. On the other hand, healing of the amputation wound should be assessed before the procedure to avoid wound healing problems, infections, and reamputations. Zimmermann et al. evaluated the use of ICG fluorescence angiography at an early postoperative time point to predict the tissue necrosis at the level of amputation. The perfusion of amputation stumps was measured with the IC-ViewSystem. In total 10 patients with critical limb ischemia and ischemic tissue loss were investigated within 72 hours after major amputation (above knee and below knee) with indocyanine green (ICG) fluorescence [144].

Strategies for neovascularization of ischemic cardiac or lower extremity tissue has been under intensive research recently. For example, gene technology has been studied to achieve therapeutic angiogenesis for peripheral arterial disease. One major problem in this investigation has been visualization and quantification of collateral growth in small animal models. The current gold standard of minimal invasive determination of blood perfusion within the hind limb of mice is the laser Doppler perfusion imaging (LDPI). However, it does not penetrate the entire limb and, thus, measures relative superficial perfusion rather than collaterals in muscle layer. Wuestenfeld et al. evaluated the applicability of the ICG angiography for the determination of hind limb perfusion in mice and compared it to LDPI. The authors suggest that ICGA is a potent tool for the quantification of collateral flow in small animal models and that LDPI shows unreliable high perfusion in the operated foot after one week indicating that it measures perfusion in the superficial skin rather than entire hind limb [145].

Lipid rich vulnerable plaques are the main cause of acute vessel occlusion in atherosclerosis. It has been recognised that ICG is a lipophilic molecule that accumulates at sites of lipid and inflammation. In animal models, it has been shown that ICG accumulates in lipid in aortic plaques and helps localise the atheromas. Furthermore, in human carotid artery specimens it has been demonstrated that ICG colocalised with lipid-rich atheroma and macrophages. Together these results suggest that ICG may be useful as a imaging agent specifically for lipid-rich and inflammatory atherosclerotic vessel lesions [146].

ICG fluorescence imaging has also been used to measure splanchnic blood flow. Leppikangas et al. studied the effects of levosimendan on systemic and splanchnic circulation during and after abdominal aortic surgery in a doubleblinded randomized study, in which 10 patients received levosimendan and 10 patients placebo. The total splanchnic blood flow was estimated by measuring the indocyanine green plasma disappearance rate (ICG-PDR) transcutaneously. Each patient was connected to an ICG finger clip, which was connected to a liver function monitor (LiMon). A $0.25 \mathrm{mg} / \mathrm{kg}$ dose of ICG was injected through a central venous line of the pulmonary artery catheter at baseline, before and during aortic clamping, and postoperatively. Levosimendan did not have a significant effect on total splanchnic perfusion in patients undergoing an elective aortic aneurysm operation [147].

Foam sclerotherapy is a widely used treatment for varicose veins. The spreading of the sclerosant is usually visualized by ultrasound. Kikuchi et al. reported the development of visualized sclerotherapy procedure using PDE. Camera images were digitized for real-time display and reviewed. Operating lights were turned off during imaging. ICG was mixed with polidocanol and air. In total, 35 patients were treated and studied. In all patients, sclerosant spreading was seen as excellent, and no side effects from ICG were observed [148].

4.5. Oncology and Sentinel Lymph Node Harvesting. The pioneering work of Chen et al. using a rat model shows that ICG injected in cancer tumor can be used in laser assisted photothermal- and photoimmuno-therapies [149, 150]. The first clinical trials of this kind of therapy have recently done successfully by the same group [151].

Lymph nodes are the initial site for metastases for most cancers. According to surgical principles, all cancer tissue within the primary tumour and metastatic lymph nodes should be removed during the surgical operation in order to achieve a complete and potentially curative resection. The sentinel node is the lymph node that receives the first lymph flow from a malignant tumour, and universally it is the first station, where a potential dissemination of malignant disease can be identified [152]. A real problem in cancer surgery is that the lymph nodes are difficult to harvest during operation. Currently radioactive technetium- $99 \mathrm{~m}$ isotope labeling is used to detect lymph nodes. This may be replaced by ICG NIR imaging. This attractive method to facilitate the visualisation of lymphatic vessels, sentinel nodes, and metastatic lymph nodes has been introduced by Lim and Soter [153]. Here, ICG is injected under the skin from where it flows via lymph circulation to lymph nodes revealing them when lit with excitation light $[92,98$, 154]. More recently, Kim et al. have used a dual-modality lymph node mapping to detect sentinel lymph nodes in rats, combining photoacoustic and fluorescence imaging [155]. Ito and colleagues utilised sentinel node navigation based on ICG in patients who were diagnosed to have lung cancer [156]. They concluded sentinel node navigation using ICG in lung cancer to be feasible, but some modifications will be necessary before the method can be clinically applied. Following the clinical approval of ICG for cutaneous Kaposi's sarcoma and cutaneous metastases of a rectal carcinoma they have been treated successfully [157-159]. Crane et al. have 
very recently used ICG in transcutaneous SLN detection in vulvar cancer patients [160] and Inoue et al. for identification of lymphatic pathway involved in the spreading of prostate cancer [161].

Albumin affects ICG fluorescence efficiency. Therefore in some studies albumin, usually human serum albumin (HSA), has been mixed with ICG in order to increase the fluorescence efficiency and thus sensitivity of ICG in SLN detection. However, a very recent randomised, double-blind comparison of ICG with and without HSA seems to indicate that there is no increase in sensitivity at least in the case of breast cancer [162].

We believe that the possibility to identify lymphatic vessels and appropriate lymph nodes in the operating room during surgery would yield marked benefits in terms of completeness of surgical resection and perioperative evaluation of potential dissemination of a malignant and deadly disease.

4.5.1. Lymphography. The lymphatic system is vital for many physiological processes, including immune reactions, and the maintenance of body fluid and chemical balances. The lack of noninvasive methods to monitor lymphatic pumping dynamics has been perhaps the most important reason for keeping the role of lymphatics modest in the clinical setting.

Unno et al. have recently shown how ICGI can be used in a minimally invasive method of monitoring human lymphatic pumping with a commercially available device and a custom-made transparent sphygmomanometer [163].

4.6. Liver Surgery and Laparoscopy. ICG has been used for many years as a test for hepatic function and to measure hepatic blood flow in humans and different animal species [164]. In these tests, ICG clearance has mainly been assessed by its blood clearance curve $[165,166]$. There are a limited number of studies evaluating the hepatic blood flow and liver function by direct ICG clearance using NIRS in healthy rabbits and rabbits with surgically reduced hepatic blood flow or experimentally induced liver cirrhosis [167]. The studies showed that measurement of ICG clearance by NIRS is promising for the assessment of liver dysfunction and may have applications in hepatic surgery and transplantation. Furthermore, the technique reflects the reduced liver blood flow and perfusion in liver cirrhosis more accurately than the previously used peripheral blood ICG clearance. The ICG excretion determined by NIRS correlated with the degree of parenchymal liver dysfunction [167]. A recent study in humans revealed that the measurement of hepatic ICG uptake by NIRS could become a valuable tool for assessing the indication for venous reconstruction in living donor liver transplantation and/or split donor liver transplantation. All studies mentioned show the potentials of NIRS-based determination of ICG clearance for the assessment of parenchymal liver function and perfusion. However, the absorption intensity of the liver after ICG injection by NIRS was determined in all studies by attaching NIRS sensors to the liver during laparotomy [168]. Injection of ICG via portal vein and subsequent imaging can be used to intraoperationally visualise liver segments and subsegments [169].
Ishizawa et al. have used ICG in a routine liver test preoperationally, after which a prototype ICG fluorescence imager was used to detect hepatocellular carcinoma intraoperationally during a laparoscopic hepatectomy [93].

In 2009, two clinical studies revealed that real-time ICGfluorescent imaging enabled the highly sensitive identification of small, grossly unidentifiable liver cancers. This led to an enhanced accuracy of operative staging and liver resection. Currently, indocyanine green fluorescence imaging navigation is considered to be a promising tool for clinical exploration for hepatocellular carcinoma and for routine intraoperative imaging during hepatic resection [170, 171].

Sentinel lymph node detection has been one main application of ICG in laparoscopic studies including early gastric cancer treatment and gastrectomy [172-177]. Harada et al. compared conventional and ICG-based laparoscopic sentinel node mapping for colorectal cancer and conclude that the latter is superior to the former [178]. Miyashiro et al. have recently applied a prototype ICG laparoscopic system [179] to detect sentinel nodes in gastric cancer surgery [180]. Jeong et al. have developed a new NOTES procedure for laparoscopic sentinel lymph node dissection of the stomach with ICG marking using a pig model [181].

Note, that $\mathrm{CO}_{2}$ pneumoperitoneum used in laparoscopic operations, which decreases liver blood flow, also increases ICG half-life [182].

Laparoscopic Cholecystectomy. While ICG is rapidly excreted via the bile duct, it is most natural to apply ICG intraoperationally to aid bile examination and operations [183]. Indeed, several groups have recently showed how ICG can be used to aid both open and laparoscopic cholecystectomy [184-189]. An earlier work by Ikeda et al. uses ICG for monitoring primary sclerosing cholangitis [190].

4.7. Reconstructive Microsurgery. For the past 20 years, the use of different fasciocutaneous perforator flaps has become popular in the field of reconstructive plastic surgery. Flaps that are over $15 \times 30 \mathrm{~cm}$ in size can be raised on a single perforating artery and its concomitant vein. These flaps have been used to reconstruct a wide range of different tissue defects. Even a partial loss of the flap can lead to the total failure of the reconstruction. In perforator flaps, the perfusion of the most distal parts of the flaps is often problematic. Recently, several reports have shown the feasibility of ICG angiography in the intraoperative assessment of flap viability [191-199]. ICG angiography has also been used to assess the patency of the microvascular anastomosis intraoperatively, and the intrinsic transit time of the flap circulation with promising results [200-202]. In the preoperative planning of perforator flap reconstructions, ICG angiography has been used to detect suitable perforator vessels, which facilitates the design of the flap [203]. After free tissue transfer surgery, the flap circulation has to be carefully followed. Although other methods exist for continuous flap monitoring, ICG angiography may be helpful in the early postoperative phase for the detection of anastomotic thrombosis, when flap survival is in doubt [144, 202, 204-207]. In large axial or random pattern flaps, ICG angiography can be 
used intraoperatively for deciding the need for a delay procedure to ensure flap survival [205, 208-210]. In breast reconstructions, transverse abdominal flaps can be raised on superficial vessels alone (SIEA-flap) or more commonly using the deep inferior epigastric perforator flap (DIEP or TRAM flaps). The choice is often made intraoperatively, and ICG angiography is found to be a beneficial tool in the decision making [211-216]. There has also been interest in using ICG angiography in evaluation of tissue viability, especially in traumatic degloving wounds and burns. This evidence is sparse, though, and further research is needed [191, 217-219].

Intraoperative assessment of flood flow is important for successful transplantations. Hoffmann et al. have used laser-assisted ICGA (IC-VIEW) for successful intraoperative assessment of kidney allograft perfusion in 10 consecutive de novo renal transplantations [220]. Sawada et al. have recently used ICGA by PDE for intraoperative assessment of renal vasculature after revascularisation of a transplanted kidney [221]. Mizuno and Isaji have used ICG-injected intrabiliary for donor liver bile duct imaging [222].

4.8. Other Clinical Applications. In addition to the above surgical applications, the clinical application of ICG include such topics as brain imaging and hemodynamics [223, 224], rheumatoid arthritis [225-227], burns and other trauma [228], and muscle perfusion [229].

Due to the absorption at wavelengths under $800 \mathrm{~nm}$, ICG can be used with a suitable intense light source, typically a laser, as an ingredient of a tissue solder like albumin, which readily bounds to ICG [230-238]. ICG-based tissue soldering could be one method for making surgery more automatic as laser-based ophthalmic surgery is already today.

4.8.1. Photodynamic and Photothermal Therapy. When an ICG molecule is excited, it can further transfer energy to other molecules. When exciting oxygen, ICG turns out to be a photodynamic therapy agent. In principle, for example, after having been used to reveal lymph nodes a strong illumination with NIR light could be used to destroy metastatic nodes. ICG binds easily to tissue even at high concentrations, and the visual change in colour from green to orange is manifested by the wavelength shift in reflectance peak. ICG has been used in vitro laser-assisted fat cell destruction, which might give a new optics-based procedure for cosmetic surgery [239].

Similarly, ICGA can be used as a light-activated antibacterial agent (LAAA), for example, in wound healing [234], or treating chronic rhinosinusitis [240] with near-infrared laser illumination (NILI). It was shown recently that the photodynamic effect can be used for acne treatment [241243]. Nevertheless, many problems have to be solved in order to design optimal technology for acne treatment without side effects.

Through intense light (laser) irradiation a number of new effects can be provided, which lead to more effective bacteria killing and controllable cell destruction and/or inhibition of excessive synthesis of sebum in sebocytes, like the localised photodynamic effect based on the appropriate concentration of the suitable exogenous dye incorporated into hair follicle or any other skin appendages. The indocyanine green is one of the prospective exogenous dyes for soft photodynamic treatment (PDT).

4.9. Dyeing. Finally, as ICG is a dye it can be naturally used for tattooing, labeling, and similar tasks [244-249]. A relatively early work demonstrates how ICG can be used to stain caries lesions for the further removal of lesion by a laser by the help of the high light absorption of ICG at the excitation wavelength [250]. Recently, Kitai et al. have used ICG for monitoring of perineal wound contamination in abdominoperineal resection [251].

Because hepatocytes handle ICG in the liver, ICG can be used also to monitor differentiation of mouse embryonic stem cells into hepatocytes [252, 253].

\section{Conclusions}

In this work, we have reviewed well over 200 papers describing the development and use of the fluorescing contrast agent indocyanine green in clinical, mainly surgical, applications. Many interesting works had to be omitted simply due to space limitations. However, it is hoped that we have succeeded in collecting most of the key publications for giving an overview of the indocyanine green fluorescence technology and its most important emerging clinical application.

Many new clinical applications of ICG and ICG angiography are just emerging and more are definitely expected to appear in the near future; thus, it is obvious that much more research is still needed in order to fully realise all the potential of this relatively simple optical technology. The obvious fields of further engineering research include

(i) image processing of ICG and ICGA information, also in real-time (video and stereo) [143, 239, 254, 255],

(ii) capillary circulation monitoring and perfusion dynamics imaging [256-262],

(iii) combining ICG and other imaging modalities like visual, CT, MRI, and PET [263-266],

(iv) combining ICG and ICGA with dermal imaging methods [110],

(v) deeper imaging (optical tomography) [261, 267],

(vi) optical imaging device development (laparoscopy) and optimisation, hands-free [4, 268, 269],

(vii) development of new derivatives of ICG for more specific imaging modes,

(viii) increasing the quantum efficiency of ICG by, for example, metallic nanoparticles [270, 271],

(ix) micro- and nano-encapsulation of ICG for nonangiography applications [76, 272],

(x) extraction of spectral information and chemometry (multispectral imaging) [273], and

(xi) integration of ICG imaging to robotic-assisted surgery [274]. 
In a clinical setting, ICG is a new and unique method in imaging of the lymphatic circulatory system and thus offers both challenges and the potential for totally new clinical applications [27].

\section{Abbreviations}

\begin{tabular}{|c|c|}
\hline 3D: & Three-dimensional \\
\hline ALT: & Anterolateral thigh flap \\
\hline ASA: & $\begin{array}{l}\text { American Society of Anesthesiology Patient } \\
\text { Classification Status }\end{array}$ \\
\hline AVM: & Arteriovenous malformation \\
\hline BP: & Blood pressure \\
\hline BSA: & Bovine serum albumin \\
\hline BSS: & Balanced salt solution \\
\hline CABG: & Coronary artery bypass grafting \\
\hline CCD: & Charge coupled device (camera) \\
\hline CEA: & Carotid end arterectomy \\
\hline DSA: & Digital subtraction angiography \\
\hline EPR: & Enhanced permeability and retention \\
\hline FDA: & (US) Food and drug administration \\
\hline FLIM: & Fluorescence Life-time imaging microscopy \\
\hline FOV: & Field of view FT fluorescence tomography \\
\hline HDL: & High-density lipoprotein \\
\hline HAS: & Human serum albumin \\
\hline HUCH: & Helsinki University Central Hospital \\
\hline ICA: & Independent component analysis \\
\hline ICCD: & Image-intensified CCD \\
\hline ICG: & Indocyanine green \\
\hline ICG-PDR: & ICG plasma disappearance rate \\
\hline ICG-VA: & ICG video angiography \\
\hline ICGA: & ICG angiography \\
\hline ICGI: & ICG imaging \\
\hline ICG-PDR: & ICG plasma disappearance rate \\
\hline ICU: & Intensive care unit \\
\hline IfCG: & Infracyanine green \\
\hline IREE: & Infrared-ray electronic endoscopy \\
\hline ISPI: & In situ photoImmunotherapy \\
\hline LAAA: & Light-activated antimicrobial agents \\
\hline LDL: & Low-density lipoprotein \\
\hline LDPI: & Laser Doppler perfusion imaging \\
\hline LED: & Light-emitting diode \\
\hline LIFE: & $\begin{array}{l}\text { Laparoscopic intragastric full-thickness } \\
\text { excision }\end{array}$ \\
\hline MICAD: & $\begin{array}{l}\text { Molecular Imaging and Contrast Agent } \\
\text { Database }\end{array}$ \\
\hline MRI: & Magnetic resonance imaging \\
\hline NILI: & Near-infrared laser illumination \\
\hline NIR: & Near-infra red \\
\hline NIRF: & NIR fluorescence \\
\hline NIRS: & NIR spectroscopy \\
\hline NOTES: & $\begin{array}{l}\text { Natural orifice translumenal endoscopic } \\
\text { surgery }\end{array}$ \\
\hline OCT: & Optical coherence tomography \\
\hline PAD: & Peripheral arterial disease \\
\hline PAOD: & Peripheral arterial occlusive disease \\
\hline PASP: & Sodium polyaspartate \\
\hline PBS: & Phosphate-buffered saline \\
\hline PDT: & Photodynamic therapy \\
\hline
\end{tabular}

PPG: Photoplethysmography

PPT: Photothermal therapy

PV: $\quad$ Peripheral vasculature

PVD: PV disease

QD: Quantum dot

RBC: Red blood cell

RGB: Red green blue

RP: Raynaud phenomenon

RPE: Retinal pigment epithelium

SLD: Superluminescent diode

SLN: Sentinel lymph node

SLNB: Sentinel lymph node biopsy

SNNS: Sentinel node navigation surgery

SNR: Signal-to-noise ratio

TTFM: Transit-time flowmetry.

\section{Acknowledgments}

The FIELD NIRce project and especially Professor Paul Geladi at SLU University are acknowledged for both their economic and scientific support (J. T. Alander and P. Välisuo) of this paper and NIR spectroscopy development in general. This work is a part of the FIELD NIRce project which is a subproject of Bothnia-Atlantica. A preliminary version of this work was presented at the ICNIR 2009 Conference by one of the authors (J. T. Alander) [275]. V. Tuchin was supported by grants CRDF (RUB1-2932-SR-08), RFBR(10-07-00526-a). PHOTONICS4LIFE of FP7 (224014), RF Ministry of Edu. and Sci. (1.4.09, 2.1.1/4989, 2.2.1.1/2950), RF Govern. contracts (02.740.11.0484, 02.740.11.0770, 02.740.11.0879); FDiPro, Tekes Program, Finland (3081/31/2010). MD Anders Albäck at $\mathrm{HUCH}$ is acknowledged for his many helpful comments on vascular surgery, MD Hiroaki Terasaki vising $\mathrm{HUCH}$ from Tokyo Medical and Dental University Hospital of Medicine for his great help with angiography by the PDE equipment, and PhD Outi Villet at $\mathrm{HUCH}$ is acknowledged for her help with ICGA rat coronary imaging.

\section{References}

[1] Q. Wu, F. A. Merchant, and K. R. Castleman, Eds., Microscope Image Processing, Academic Press, New York, NY, USA, 2008.

[2] M. Choi, K. Choi, S.-W. Ryu, J. Lee, and C. Choi, "Dynamic fluorescence imaging for multiparametric measurement of tumor vasculature," Journal of Biomedical Optics, vol. 16, no. 4, Article ID 046008, 2011.

[3] E. M. Sevick-Muraca, J. P. Houston, and M. Gurfinkel, "Fluorescence-enhanced, near infrared diagnostic imaging with contrast agents," Current Opinion in Chemical Biology, vol. 6, no. 5, pp. 642-650, 2002.

[4] Y. Liu, A. Q. Bauer, W. J. Akers et al., "Hands-free, wireless goggles for near-infrared fluorescence and real-time imageguided surgery," Surgery, vol. 149, no. 5, pp. 689-698, 2011.

[5] J. T. Alander, "Processing of indocyanine green fluorescence images," In Preparation.

[6] Ó. G. Björnsson, R. Murphy, and V. S. Chadwick, "Physicochemical studies of indocyanine green (ICG): absorbance/ concentration relationship, $\mathrm{pH}$ tolerance and assay precision 
in various solvents," Experientia, vol. 38, no. 12, pp. 14411442, 1982.

[7] E. Engel, R. Schraml, T. Maisch et al., "Light-induced decomposition of indocyanine green," Investigative Ophthalmology and Visual Science, vol. 49, no. 5, pp. 1777-1783, 2008.

[8] K. Kogure and E. Choromokos, "Infrared absorption angiography," Journal of Applied Physiology, vol. 26, no. 1, pp. 154157, 1969.

[9] R. W. Flower, "Injection technique for indocyanine green and sodium fluorescein dye angiography of the eye," Investigative Ophthalmology, vol. 12, no. 12, pp. 881-895, 1973.

[10] J. V. Frangioni, "In vivo near-infrared fluorescence imaging," Current Opinion in Chemical Biology, vol. 7, no. 5, pp. 626634, 2003.

[11] C. L. Amiot, S. Xu, S. Liang, L. Pan, and J. X. Zhao, "Near-infrared fluorescent materials for sensing of biological targets," Sensors, vol. 8, no. 5, pp. 3082-3105, 2008.

[12] P. L. Choyke, R. Alford, H. M. Simpson et al., "Toxicity of organic fluorophores used in molecular imaging: literature review," Molecular Imaging, vol. 8, no. 6, pp. 341-354, 2009.

[13] L. A. Yannuzzi, "Indocyanine green angiography: a perspective on use in the clinical setting," American Journal of Ophthalmology, vol. 151, no. 5, pp. 745-751, 2011.

[14] A. Agarwal, Ed., Fundus Fluorescein and Indocyanine Green Angiography, A Textbook and Atlas, SLACK Incorporated, Thorofare, NJ, USA, 2008.

[15] S. L. Owens, "Indocyanine green angiography," British Journal of Ophthalmology, vol. 80, no. 3, pp. 263-266, 1996.

[16] D. Stanescu-Segall and T. L. Jackson, "Vital staining with indocyanine green: a review of the clinical and experimental studies relating to safety," Eye, vol. 23, no. 3, pp. 504-518, 2009.

[17] C. H. Meyer, R. A. Oechsler, and E. B. Rodrigues, "Historical considerations in applying vital dyes in vitreoretinal surgery: from early experiments to advanced chromovitrectomy," Expert Reviews in Ophthalmology, vol. 2, no. 1, pp. 71-77, 2007.

[18] A. Godavarty, M. J. Eppstein, C. Zhang et al., "Fluorescenceenhanced optical imaging in large tissue volumes using a gain-modulated ICCD camera," Physics in Medicine and Biology, vol. 48, no. 12, pp. 1701-1720, 2003.

[19] A. Kortgen, P. Recknagel, and M. Bauer, "How to assess liver function?" Current Opinion in Critical Care, vol. 16, no. 2, pp. 136-141, 2010.

[20] G. Paumgartner, "Biliary physiology and disease: reflections of a physician-scientist," Hepatology, vol. 51, no. 4, pp. 1095$1106,2010$.

[21] J. P. Houston, Near infrared fluorescent optical lymphography for cancer diagnostics, Ph.D. thesis, Texas A\&M University, Chemical Engineering, College Station, Tex, USA, 2005.

[22] E. A. te Velde, T. Veerman, V. Subramaniam, and T. Ruers, "The use of fluorescent dyes and probes in surgical oncology," European Journal of Surgical Oncology, vol. 36, no. 1, pp. 6$15,2010$.

[23] B. E. Schaafsma, J. S.D. Mieog, M. Hutteman et al., "The clinical use of indocyanine green as a near-infrared fluorescent contrast agent for image-guided oncologic surgery," Journal of Surgical Oncology, vol. 104, no. 3, pp. 323-332, 2011.

[24] K. Polom, D. Murawa, Y.-S. Rho, P. Nowaczyk, M. Hünerbein, and P. Murawa, "Current trends and emerging future of indocyanine green usage in surgery and oncology: a literature review," Cancer, vol. 117, no. 21, pp. 4812-4822, 2011.
[25] S. Luo, E. Zhang, Y. Su, T. Cheng, and C. Shi, "A review of NIR dyes in cancer targeting and imaging," Biomaterials, vol. 32, no. 29, pp. 7127-7138, 2011.

[26] M. Kaiser, A. Yafi, M. Cinat, B. Choi, and A. J. Durkin, "Noninvasive assessment of burn wound severity using optical technology: a review of current and future modalities," Burns, vol. 37, no. 3, pp. 377-386, 2011.

[27] M. V. Marshall, J. C. Rasmussen, I.-V. Tan et al., "Nearinfrared fluorescent imaging in humans with indocyanine green: a review and update," The Open Surgical Oncological Journal, vol. 2, pp. 12-25, 2010.

[28] MICAD, Molecular Imaging and Contrast Agent Database (MICAD), National Library of Medicine (NCBIUS), Bethesda, Md, USA, 2004.

[29] B. Yuan, N. G. Chen, and Q. Zhu, "Emission and absorption properties of indocyanine green in intralipid solution," Journal of Biomedical Optics, vol. 9, no. 3, pp. 497-503, 2004.

[30] M. Miwa and T. Shikayama, "ICG fluorescence imaging and its medical applications," in Proceedings of the 2008 International Conference on Optical Instruments and Technology: Optoelectronic Measurement Technology and Applications, S. Ye, G. Zhang, and J. Ni, Eds., vol. SPIE-7160, The International Society for Optical Engineering, Beijing, China, 2009.

[31] Z. Zhang, M. Y. Berezin, J. L. F. Kao, A. D’Avignon, M. Bai, and S. Achilefu, "Near-infrared dichromic fluorescent carbocyanine molecules," Angewandte Chemie, vol. 47, no. 19, pp. 3584-3587, 2008.

[32] J. V. Frangioni, "New technologies for human cancer imaging," Journal of Clinical Oncology, vol. 26, no. 24, pp. 40124021, 2008.

[33] A. Mishra, R. K. Behera, P. K. Behera, B. K. Mishra, and G. B. Behera, "Cyanines during the 1990s: a review," Chemical Reviews, vol. 100, no. 6, pp. 1973-2011, 2000.

[34] R. Rajagopalan, P. Uetrecht, J. E. Bugaj, S. A. Achilefu, and R. B. Dorshow, "Stabilization of the optical tracer agent indocyanine green using noncovalent interactions," Photochemistry and Photobiology, vol. 71, no. 3, pp. 347-350, 2000.

[35] J.-M. I. Maarek, D. P. Holschneider, and J. Harimoto, "Fluorescence of indocyanine green in blood: intensity dependence on concentration and stabilization with sodium polyaspartate," Journal of Photochemistry and Photobiology B, vol. 65, no. 2-3, pp. 157-164, 2001.

[36] R. D. Hall and C. F. Chignell, "Steady-state near-infrared detection of singlet molecular oxygen: a Stern-Volmer quenching experiment with sodium azide," Photochemistry and Photobiology, vol. 45, no. 4, pp. 459-464, 1987.

[37] F. Rotermund, R. Weigand, W. Holzer, M. Wittmann, and A. Penzkofer, "Fluorescence spectroscopic analysis of indocyanine green J aggregates in water," Journal of Photochemistry and Photobiology A, vol. 110, no. 1, pp. 75-78, 1997.

[38] M. L. J. Landsman, G. Kwant, G. A. Mook, and W. G. Zijlstra, "Light absorbing properties, stability, and spectral stabilization of indocyanine green," Journal of Applied Physiology, vol. 40, no. 4, pp. 575-583, 1976.

[39] S. Fickweiler, R. M. Szeimies, W. Bäumler et al., "Indocyanine green: intracellular uptake and phototherapeutic effects In vitro," Journal of Photochemistry and Photobiology B, vol. 38, no. 2-3, pp. 178-183, 1997.

[40] K. Tokuda, C. F. Zorumski, and Y. Izumi, "Involvement of illumination in indocyanine green toxicity after its washout in the ex vivo rat retina," Retina, vol. 29, no. 3, pp. 371-379, 2009.

[41] T. Sato, M. Ito, M. Ishida, and Y. Karasawa, "Phototoxicity of indocyanine green under continuous fluorescent lamp 
illumination and its prevention by blocking red light on cultured müller cells," Investigative Ophthalmology and Visual Science, vol. 51, no. 8, pp. 4337-4345, 2010.

[42] K. J. Baker, "Binding of sulfobromophthalein (BSP) sodium and indocyanine green (ICG) by plasma alpha-1 lipoproteins," Proceedings of the Society for Experimental Biology and Medicine, vol. 122, no. 4, pp. 957-963, 1966.

[43] K. Kamisaka, Y. Yatsuji, H. Yamada, and H. Kameda, "The binding of indocyanine green and other organic anions to serum proteins in liver diseases," Clinica Chimica Acta, vol. 53, no. 2, pp. 255-264, 1974.

[44] S. Yoneya, T. Saito, Y. Komatsu, I. Koyama, K. Takahashi, and J. Duvoll-Young, "Binding properties of indocyanine green in human blood," Investigative Ophthalmology and Visual Science, vol. 39, no. 7, pp. 1286-1290, 1998.

[45] V. I. Kochubey, T. V. Kulyabina, V. V. Tuchin, and G. B. Altshuler, "Spectral characteristics of indocyanine green upon its interaction with biological tissues," Optics and Spectroscopy, vol. 99, no. 4, pp. 560-566, 2005.

[46] C. Ciamberlini, V. Guarnieri, G. Longobardi, P. Poggi, M. C. Donati, and G. Panzardi, "Indocyanine green videoangiography using cooled charge-coupled devices in central serous choroidopathy," Journal of Biomedical Optics, vol. 2, no. 2, pp. 218-225, 1997.

[47] R. A. Weersink, J. E. Hayward, K. R. Diamond, and M. S. Patterson, "Accuracy of noninvasive In vivo measurements of photosensitizer uptake based on a diffusion model of reflectance spectroscopy," Photochemistry and Photobiology, vol. 66, no. 3, pp. 326-335, 1997.

[48] E. A. Genina, A. N. Bashkatov, V. I. Kochubei, V. V. Tuchin, and G. B. Altshuler, "The interaction of Indocyanine Green dye with the human epidermis studied In vivo," Technical Physics Letters, vol. 27, no. 7, pp. 602-604, 2001.

[49] S. Gioux, S. J. Lomnes, H. S. Choi, and J. V. Frangioni, "Lowfrequency wide-field fluorescence lifetime imaging using a high-power near-infrared light-emitting diode light source," Journal of Biomedical Optics, vol. 15, no. 2, p. 026005, 2010.

[50] H. Ohkubo, H. Musha, and K. Okuda, "Effects of caloric restriction on the kinetics of indocyanine green in patients with liver diseases and in the rat," American Journal of Digestive Diseases, vol. 23, no. 11, pp. 1017-1024, 1978.

[51] N. Tanaka, K. Kanai, and T. Oda, "On the pathological modification of serum high density lipoproteins in liver diseases. An alteration in the binding mode of indocyanine green with human serum proteins," Clinica Chimica Acta, vol. 49, no. 3, pp. 333-340, 1973.

[52] M. Reekers, M. J. G. Simon, F. Boer et al., "Pulse dye densitometry and indocyanine green plasma disappearance in ASA physical status I-II patients," Anesthesia and Analgesia, vol. 110, no. 2, pp. 466-472, 2010.

[53] G. Paumgartner, "The handling of indocyanine green by the liver," Schweizerische Medizinische Wochenschrift, vol. 105, no. 17, 1975.

[54] J. F. Kiilgaard, M. H. Nissen, and M. la Cour, "An isotonic preparation of $1 \mathrm{mg} / \mathrm{ml}$ indocyanine green is not toxic to hyperconfluent ARPE19 cells, even after prolonged exposure," Acta Ophthalmologica Scandinavica, vol. 84, no. 1, pp. 42-46, 2006.

[55] T. L. Jackson, B. Vote, B. C. Knight, A. El-Amir, M. R. Stanford, and J. Marshall, "Safety testing of infracyanine green using retinal pigment epithelium and glial cell cultures," Investigative Ophthalmology and Visual Science, vol. 45, no. 10, pp. 3697-3703, 2004.
[56] F. M. Penha, M. Maia, M. E. Farah et al., "Morphologic and clinical effects of subretinal injection of indocyanine green and infracyanine green in rabbits," Journal of Ocular Pharmacology and Therapeutics, vol. 24, no. 1, pp. 52-61, 2008.

[57] H. F. Yam, A. K. H. Kwok, K. P. Chan et al., "Effect of indocyanine green and illumination on gene expression in human retinal pigment epithelial cells," Investigative Ophthalmology and Visual Science, vol. 44, no. 1, pp. 370-377, 2003.

[58] M. V. Marshall, D. Draney, E. M. Sevick-Muraca, and D. M. Olive, "Single-dose intravenous toxicity study of IRDye 800CW in sprague-dawley Rats," Molecular Imaging and Biology, vol. 12, no. 6, pp. 583-594, 2010.

[59] F. B. Dietz and R. A. Jaffe, "Indocyanine green: evidence of neurotoxicity in spinal root axons," Anesthesiology, vol. 98, no. 2, pp. 516-520, 2003.

[60] S.-L. Hsu, Y. H. Kao, and W. C. Wu, "Effect of indocyanine green on the growth and viability of cultured human retinal pigment epithelial cells," Journal of Ocular Pharmacology and Therapeutics, vol. 20, no. 4, pp. 353-362, 2004.

[61] A. A. Chang, M. Zhu, and F. Billson, "The interaction of indocyanine green with human retinal pigment epithelium," Investigative Ophthalmology and Visual Science, vol. 46, no. 4, pp. 1463-1467, 2005.

[62] K. Kuroda, H. Kinouchi, K. Kanemaru, T. Wakai, N. Senbokuya, and T. Horikoshi, "Indocyanine green videoangiography to detect aneurysm and related vascular structures buried in subarachnoid clots: case report," Journal of Neurosurgery, vol. 114, no. 4, pp. 1054-1056, 2011.

[63] E. S. Nussbaum, A. Defillo, and L. Nussbaum, "The use of indocyanine green videoangiography to optimize the dural opening for intracranial parasagittal lesions," Neurosurgery. In press.

[64] S. Homina, T. Fukunaga, and A. Kagaya, "Influence of adipose tissue thickness on near infrared spectroscopic signals in the measurement of human muscle," Journal of Biomedical Optics, vol. 1, no. 4, pp. 418-424, 1996.

[65] V. V. Tuchin, Tissue Optics: Light Scattering Methods and Instruments for Medical Diagnosis, The International Society for Optics and Photonics (SPIE), Bellingham, Wash, USA, 2007.

[66] J. Chen, I. R. Corbin, H. Li, W. Cao, J. D. Glickson, and G. Zheng, "Ligand conjugated low-density lipoprotein nanoparticles for enhanced optical cancer imaging In vivo," Journal of the American Chemical Society, vol. 129, no. 18, pp. 57985799, 2007.

[67] H. Stepp, H. Schachenmayr, A. Ehrhardt, W. Göbel, S. Zhorzel, and C. S. Betz, "Endoscopic ICG perfusion imaging for flap transplants: technical development," in Photonic Therapeutics and Diagnostics VI, N. Kollias, Ed., vol. SPIE7548, The International Society for Optical Engineering, Bellingham, Wash, USA, 2010.

[68] G. Sun, M. Y. Berezin, J. Fan et al., "Bright fluorescent nanoparticles for developing potential optical imaging contrast agents," Nanoscale, vol. 2, no. 4, pp. 548-558, 2010.

[69] V. B. Rodriguez, S. M. Henry, A. S. Hoffman, P. S. Stayton, X. Li, and S. H. Pun, "Encapsulation and stabilization of indocyanine green within poly(styrene-alt-maleic anhydride) block-poly(styrene) micelles for near-infrared imaging," Journal of Biomedical Optics, vol. 13, no. 1, p. 014025 , 2008.

[70] A. K. Kirchherr, A. Briel, and K. Mäder, "Stabilization of indocyanine green by encapsulation within micellar systems," Molecular Pharmaceutics, vol. 6, no. 2, pp. 480-491, 2009. 
[71] M. Ogawa, N. Kosaka, P. L. Choyke, and H. Kobayashi, "In vivo molecular imaging of cancer with a quenching nearinfrared fluorescent probe using conjugates of monoclonal antibodies and indocyanine green," Cancer Research, vol. 69, no. 4, pp. 1268-1272, 2009.

[72] F. Liu, D. Deng, X. Chen, Z. Qian, S. Achilefu, and Y. Gu, "Folate-polyethylene glycol conjugated near-infrared fluorescence probe with high targeting affinity and sensitivity for In vivo early tumor diagnosis," Molecular Imaging and Biology, vol. 12, no. 6, pp. 595-607, 2010.

[73] B. Ebert, B. Riefke, U. Sukowski, and K. Licha, "Cyanine dyes as contrast agents for near-infrared imaging In vivo: acute tolerance, pharmacokinetics, and fluorescence imaging," Journal of Biomedical Optics, vol. 16, no. 6, Article ID 066003, 2011.

[74] M. A. Yaseen, J. Yu, B. Jung, M. S. Wong, and B. Anvari, "Biodistribution of encapsulated indocyanine green in healthy mice," Molecular Pharmaceutics, vol. 6, no. 5, pp. 1321-1332, 2009.

[75] R. X. Xu, J. Huang, J. S. Xu et al., "Fabrication of indocyanine green encapsulated biodegradable microbubbles for structural and functional imaging of cancer," Journal of biomedical optics, vol. 14, no. 3, p. 034020, 2009.

[76] J. Yu, D. Yavier, M. A. Yaseen et al., "Self-assembly synthesis, tumor cell targeting, and photothermal cababilities of antibody-coated indocyanine green nanocapsules," Journal of the American Chemical Society, vol. 132, no. 6, pp. 1929-1938, 2010.

[77] Y. Chen, T. Jabbour, and X. Li, "Functional fluorescent nanocapsules for molecular imaging and potential targeted therapy," in Proceedings of the European Conference on Lasers and Electro-Optics (CLEO '11), pp. 1-2, IEEE, Piscataway, NJ, May 2011.

[78] M. A. Yaseen, J. Yu, M. S. Wong, and B. Anvari, "In-vivo fluorescence imaging of mammalian organs using chargeassembled mesocapsule constructs containing indocyanine green," Optics Express, vol. 16, no. 25, pp. 20577-20587, 2008.

[79] A. Makino, S. Kizaka-Kondoh, R. Yamahara et al., "Nearinfrared fluorescence tumor imaging using nanocarrier composed of poly(l-lactic acid)-block-poly(sarcosine) amphiphilic polydepsipeptide," Biomaterials, vol. 30, no. 28, pp. 5156-5160, 2009.

[80] B. M. Barth, R. Sharma, E. I. Altinoğlu et al., "Bioconjugation of calcium phosphosilicate composite nanoparticles for selective targeting of human breast and pancreatic cancers In vivo," ACS Nano, vol. 4, no. 3, pp. 1279-1287, 2010.

[81] P. Stalmans, E. J. Feron, R. Parys-Van Ginderdeuren, A. Van Lommel, G. R. J. Melles, and M. Veckeneer, "Double vital staining using trypan blue and infracyanine green in macular pucker surgery," British Journal of Ophthalmology, vol. 87, no. 6, pp. 713-716, 2003.

[82] C. Haritoglou, A. Gandorfer, M. Schaumberger, R. Tadayoni, A. Gandorfer, and A. Kampik, "Light-absorbing properties and osmolarity of indocyanine-green depending on concentration and solvent medium," Investigative Ophthalmology and Visual Science, vol. 44, no. 6, pp. 2722-2729, 2003.

[83] J. V. Hajnal, D. L. G. Hill, and D. J. Hawkes, Eds., Medical Image Registration, CRC Press, Boca Raton, Fla, USA, 2001.

[84] C.-L. Tsai, S.-T. Huang, K.-S. Lin, and S.-J. Chen, "Robust pairwise registration for images of indocyanine-green angiographic sequences," in Proceedings of the 10th International Symposium on Pervasive Systems, Algorithms, and Networks, pp. 394-399, IEEE Computer Society, 2009.
[85] J. Reichman, Handbook of Optical Filters for Fluorescence Microscopy, Chroma Technology Corp., Brattleboro, Vt, USA, 2000.

[86] A. M. De Grand and J. V. Frangioni, "An Operational Near-Infrared Fluorescence Imaging System Prototype for Large Animal Surgery," Technology in Cancer Research and Treatment, vol. 2, no. 6, pp. 553-562, 2003.

[87] S. Hettige and D. Walsh, "Indocyanine green video-angiography as an aid to surgical treatment of spinal dural arteriovenous fistulae," Acta Neurochirurgica, vol. 152, no. 3, pp. 533-536, 2010.

[88] A. Raabe, J. Beck, R. Gerlach et al., "Near-infrared indocyanine green video angiography: a new method for intraoperative assessment of vascular flow," Neurosurgery, vol. 52, no. 1, pp. 132-139, 2003.

[89] R. Meier, S. Boddington, C. Krug et al., "Detection of postoperative granulation tissue with an ICG-enhanced integrated OI-/X-ray system," Journal of Translational Medicine, vol. 6, article no. 73, 2008.

[90] Y. Kang, M. Choi, J. Lee, G. Y. Koh, K. Kwon, and C. Choi, "Quantitative analysis of peripheral tissue perfusion using spatiotemporal molecular dynamics," PLoS ONE, vol. 4, no. 1, Article ID e4275, 2009.

[91] J. Ge, Fluorescence-enhanced optical imaging on three-dimensional phantoms using a hand-held probe based frequencydomain intensified charge coupled device (ICCD) optical imager, Ph.D. thesis, Florida International University, Miami, Fla, USA, 2008.

[92] M. Fujiwara, T. Mizukami, A. Suzuki, and H. Fukamizu, "Sentinel lymph node detection in skin cancer patients using real-time fluorescence navigation with indocyanine green: preliminary experience," Journal of Plastic, Reconstructive and Aesthetic Surgery, vol. 62, no. 10, pp. e373-e378, 2009.

[93] T. Ishizawa, Y. Bandai, N. Harada et al., "Indocyanine greenfluorescent imaging of hepatocellular carcinoma during laparoscopic hepatectomy: an initial experience," Asian Journal of Endoscopic Surgery, vol. 3, no. 1, pp. 42-45, 2010.

[94] T. Ku, J. Lee, and C. Choi, "Cerebral blood flow imaging using timeseries analysis of indocyanine green molecular dynamics in mice," in Imaging, Manipulation, and Analysis of Biomolecules, Cells, and Tissues VIII, D. L. Farkas, D. V. Nicolau, and R. C. Leif, Eds., vol. SPIE-7568, The International Society for Optical Engineering, Bellingham, Wash, USA, 2010

[95] Y. Kawaguchi, T. Ishizawa, K. Masuda et al., "Hepatobiliary surgery guided by a novel fluorescent imaging technique for visualizing hepatic arteries, bile ducts, and liver cancers on color images," Journal of the American College of Surgeons, vol. 212, no. 6, pp. e33-e39, 2011.

[96] S. K. Singh, N. D. Desai, G. Chikazawa et al., "The Graft Imaging to Improve Patency (GRIIP) clinical trial results," Journal of Thoracic and Cardiovascular Surgery, vol. 139, no. 2, pp. 294-e1, 2010.

[97] T. Kuroiwa, Y. Kajimoto, and T. Ohta, "Development and clinical application of near-infrared surgical microscope: preliminary report," Minimally Invasive Neurosurgery, vol. 44, no. 4, pp. 240-242, 2001.

[98] C. Hirche, S. Dresel, R. Krempien, and M. Hünerbein, "Sentinel node biopsy by indocyanine green retention fluorescence detection for inguinal lymph node staging of anal cancer: preliminary experience," Annals of Surgical Oncology, vol. 17, no. 9, pp. 2357-2362, 2010.

[99] R. Inoue, M. Sawa, M. Tsujikawa, and F. Gomi, "Association between the efficacy of photodynamic therapy and 
indocyanine green angiography findings for central serous chorioretinopathy," American Journal of Ophthalmology, vol. 149, no. 3, pp. 441-446.e2, 2010.

[100] G. M. Dobre, A. Gh. Podoleanu, and R. B. Rosen, "Simultaneous optical coherence tomography-Indocyanine Green dye fluorescence imaging system for investigations of the eye's fundus," Optics Letters, vol. 30, no. 1, pp. 58-60, 2005.

[101] Y. Kang, J. Lee, K. Kwon, and C. Choi, "Application of novel dynamic optical imaging for evaluation of peripheral tissue perfusion," International Journal of Cardiology, vol. 145, no. 3, pp. e99-e101, 2010.

[102] Z. Jing, S. Ou, Y. Ban, Z. Tong, and Y. Wang, "Intraoperative assessment of anterior circulation aneurysms using the indocyanine green video angiography technique," Journal of Clinical Neuroscience, vol. 17, no. 1, pp. 26-28, 2010.

[103] Y. Tsujino, K. Mizumoto, Y. Matsuzaka, H. Niihara, and E. Morita, "Fluorescence navigation with indocyanine green for detecting sentinel nodes in extramammary Paget's disease and squamous cell carcinoma," Journal of Dermatology, vol. 36, no. 2, pp. 90-94, 2009.

[104] R. C. Benson and H. A. Kues, "Fluorescence properties of indocyanine green as related to angiography," Physics in Medicine and Biology, vol. 23, no. 1, pp. 159-163, 1978.

[105] R. Sharma, W. Wang, J. C. Rasmussen et al., "Quantitative imaging of lymph function," American Journal of PhysiologyHeart and Circulatory Physiology, vol. 292, no. 6, pp. H3109H3118, 2007.

[106] E. M. Sevick-Muraca, R. Sharma, J. C. Rasmussen et al., "Imaging of lymph flow in breast cancer patients after microdose administration of a near-infrared fluorophore: feasibility study," Radiology, vol. 246, no. 3, pp. 734-741, 2008.

[107] M. J. C. van Gemert, R. Verdaasdonk, E. G. Stassen, G. A. C. M. Schects, G. H. M. Gijsbers, and J. J. Bonnier, "Optical properties of human blood vessel wall and plague," Lasers in Surgery and Medicine, vol. 5, no. 3, pp. 235-237, 1985.

[108] T. J. Farrell, M. S. Patterson, and B. Wilson, "A diffusion theory model of spatially resolved, steady-state diffuse reflectance for the noninvasive determination of tissue optical properties In vivo," Medical Physics, vol. 19, no. 4, pp. 879-888, 1992.

[109] P. Välisuo, I. Kaartinen, V. Tuchin, and J. Alander, "New closed-form approximation for skin chromophore mapping," Journal of Biomedical Optics, vol. 16, no. 4, Article ID 046012, 2011.

[110] P. Välisuo, Photonics simulation and modelling of skin for design of spectrocutometer, Ph.D. thesis, University of Vaasa, Department of Electrical Engineering and Energy Technology, Vaasa, Finland, 2011.

[111] S. Prahl, "Optical absorption of hemoglobin," Tech. Rep., Oregon Medical Laser Center, Portland, Ore, USA, 1999.

[112] A. Roggan, M. Friebel, K. Dörschel, A. Hahn, and G. Müller, "Optical properties of circulating human blood in the wavelength range 400-2500 NM," Journal of Biomedical Optics, vol. 4, no. 1, pp. 36-46, 1999.

[113] R. Philip, A. Penzkofer, W. Bäumler, R. M. Szeimies, and C. Abels, "Absorption and fluorescence spectroscopic investigation of indocyanine green," Journal of Photochemistry and Photobiology A, vol. 96, no. 1-3, pp. 137-148, 1996.

[114] J. F. R. Ilgner, T. Kawai, T. Shibata, T. Yamazoe, and M. Westhofen, "Evaluation of stereoscopic medical video content on an autostereoscopic display for undergraduate medical education," in Stereoscopic Displays and Virtual Reality Systems XIII, A. J. Woods, Ed., vol. SPIE-6055, pp. 506-605, The
International Society for Optical Engineering, San Jose, Calif, USA, 2006.

[115] S. C. Stein, M. G. Burnett, E. L. Zager, H. A. Riina, and S. S. Sonnad, "Completion angiography for surgically treted cerebral aneurysms: an economic analysis. clinical studies," Neurosurgery, vol. 61, no. 6, pp. 1162-1169, 2007.

[116] A. Raabe, P. Nakaji, J. Beck et al., "Prospective evaluation of surgical microscope-integrated intraoperative near-infrared indocyanine green videoangiography during aneurysm surgery," Journal of Neurosurgery, vol. 103, no. 6, pp. 982-989, 2005.

[117] J. Patel, K. Marks, I. Roberts, D. Azzopardi, and A. D. Edwards, "Measurement of cerebral blood flow in newborn infants using near infrared spectroscopy with indocyanine green," Pediatric Research, vol. 43, no. 1, pp. 34-39, 1998.

[118] J. G. de Oliveira, J. Beck, V. Seifert, M. J. Teixeira, and A. Raabe, "Assessment of flow in perforating arteries during intracranial aneurysm surgery using intraoperative nearinfrared indocyanine green videoangiogaphy," Neurosurgery, vol. 61, no. 3, pp. 63-73, 2007.

[119] R. Dashti, A. Laakso, M. Niemelä, M. Porras, and J. Hernesniemi, "Microscope-integrated near-infrared indocyanine green videoangiography during surgery of intracranial aneurysms: the Helsinki experience," Surgical Neurology, vol. 71, no. 5, pp. 543-550, 2009.

[120] J. Li, Z. Lan, M. He, and C. You, "Assessment of microscopeintegrated indocyanine green angiography during intracranial aneurysm surgery: a retrospective study of 120 patients," Neurology India, vol. 57, no. 4, pp. 453-459, 2009.

[121] C.-Y. Ma, J.-X. Shi, H. -D. Wang, C.-H. Hang, H.-L. Cheng, and $\mathrm{W}$. Wu, "Intraoperative indocyanine green angiography in intracranial aneurysm surgery: microsurgical clipping and revascularization," Clinical Neurology and Neurosurgery, vol. 111, no. 10, pp. 840-846, 2009.

[122] B. D. Killory, P. Nakaji, L. F. Gonzales, F. A. Ponce, S. D. Wait, and R. F. Spetzler, "Prospective evaluation of surgical microscope-integrated intraoperative near-infrared indocyanine green angiography during cerebral arteriovenous malformation surgery," Neurosurgery, vol. 65, no. 3, pp. 456462, 2009.

[123] G. P. Colby, A. L. Coon, D. M. Sciubba, A. Bydon, P. E. Gailloud, and R. J. Tamargo, "Intraoperative indocyanine green angiography for obliteration of a spinal dural arteriovenous fistula: case report," Journal of Neurosurgery, vol. 11, no. 6, pp. 705-709, 2009.

[124] D. Hänggi, N. Etminan, and H. -J. Steiger, "The impact of microscope-integrated intraoperative near-infrared indocyanine green videoangiography on surgery of arteriovenous malformations and dural arteriovenous fistulae," Neurosurgery, vol. 67, no. 4, pp. 1094-1103, 2010.

[125] J. Woitzik, P. Horn, P. Vajkoczy, and P. Schmiedek, "Intraoperative control of extracranial-intracranial bypass patency by near-infrared indocyanine green videoangiography," Journal of Neurosurgery, vol. 102, no. 4, pp. 692-698, 2005.

[126] P. G. Peña-Tapia, A. Kemmling, M. Czabanka, P. Vajkoczy, and P. Schmiedek, "Identification of the optimal cortical target point for extracranial-intracranial bypass surgery in patients with hemodynamic cerebrovascular insufficiency," Journal of Neurosurgery, vol. 108, no. 4, pp. 655-661, 2008.

[127] M. Bruneau, E. Sauvageau, P. Nakaji et al., "Preliminary personal experiences with the application of near-infrared indocyanine green videoangiography in extracranial vertebral artery surgery," Neurosurgery, vol. 66, no. 2, pp. 305-311, 2010 . 
[128] S. Haga, S. Nagata, A. Uka, Y. Akagi, Y. Hamada, and T. Shono, "Near-infrared indocyanine green videoangiography for assessment of carotid endarterectomy," Acta Neurochirurgica, vol. 153, no. 8, pp. 1641-1644, 2011.

[129] M. A. Kamp, P. Slotty, B. Turowski et al., "Microscope integrated quantitative analysis of intra-operative indocyanine green fluorescence angiography for blood flow assessment: first experience in 30 patients," Neurosurgery. In press.

[130] C. Detter, D. Russ, A. Iffland et al., "Near-infrared fluorescence coronary angiography: a new noninvasive technology for intraoperative graft patency control," Heart Surgery Forum, vol. 5, no. 4, pp. 364-369, 2002.

[131] G. D’Ancona, H. L. Karamanoukian, M. Ricci, S. Schmid, J. Bergsland, and T. A. Salerno, "Graft revision after transit time flow measurement in off-pump coronary artery bypass grafting," European Journal of Cardio-thoracic Surgery, vol. 17, no. 3, pp. 287-293, 2000.

[132] L. Balacumaraswami and D. P. Taggart, "Intraoperative imaging techniques to assess coronary artery bypass graft patency," Annals of Thoracic Surgery, vol. 83, no. 6, pp. 2251$2257,2007$.

[133] C. Detter, S. Wipper, D. Russ et al., "Fluorescent cardiac imaging: a novel intraoperative method for quantitative assessment of myocardial perfusion during graded coronary artery stenosis," Circulation, vol. 116, no. 9, pp. 1007-1014, 2007.

[134] F. D. Rubens, M. Ruel, and S. E. Fremes, "A new and simplified method for coronary and graft imaging during CABG," Heart Surgery Forum, vol. 5, no. 2, pp. 141-144, 2002.

[135] D. P. Taggart, B. Choudhary, K. Anastasiadis, Y. AbuOmar, L. Balacumaraswami, and D. W. Pigott, "Preliminary experience with a novel intraoperative fluorescence imaging technique to evaluate the patency of bypass grafts in total arterial revascularization," Annals of Thoracic Surgery, vol. 75, no. 3, pp. 870-873, 2003.

[136] O. Reuthebuch, A. Häussler, M. Genoni et al., "Novadaq SPY: intraoperative quality assessment in off-pump coronary artery bypass grafting," Chest, vol. 125, no. 2, pp. 418-424, 2004.

[137] L. Balacumaraswami, Y. Abu-Omar, B. Choudhary, D. Pigott, and D. P. Taggart, "A comparison of transit-time flowmetry and intraoperative fluorescence imaging for assessing coronary artery bypass graft patency," Journal of Thoracic and Cardiovascular Surgery, vol. 130, no. 2, pp. 315-320, 2005.

[138] M. Takahashi, T. Ishikawa, K. Higashidani, and H. Katoh, "SPY: an innovative intra-operative imaging system to evaluate graft patency during off-pump coronary artery bypass grafting," Interactive Cardiovascular and Thoracic Surgery, vol. 3, no. 3, pp. 479-483, 2004.

[139] N. D. Desai, S. Miwa, D. Kodama et al., "Improving the quality of coronary bypass surgery with intraoperative angiography: validation of a new technique," Journal of the American College of Cardiology, vol. 46, no. 8, pp. 1521-1525, 2005.

[140] N. D. Desai, Assessment of operative strategies to improve coronary bypass graft patency, Ph.D. thesis, University of Toronto, Toronto, Canada, 2008.

[141] N. Unno, K. Inuzuka, M. Suzuki et al., "Preliminary experience with a novel fluorescence lymphography using indocyanine green in patients with secondary lymphedema," Journal of Vascular Surgery, vol. 45, no. 5, pp. 1016-1021, 2007.

[142] Y. Kang, J. Lee, K. Kwon, and C. Choi, "Dynamic fluorescence imaging of indocyanine green for eable and sensitive diagnosis of peripheral vascular insufficiency," Microvascular Research, vol. 80, no. 3, pp. 552-555, 2010.

[143] Y. Kang, J. Lee, Y. An, J. Jeon, and C. Choi, "Segmental analysis of indocyanine green pharmacokinetics for the reliable diagnosis of functional vascular insufficiency," Journal of Biomedical Optics, vol. 16, no. 3, Article ID 030504, 2011.

[144] A. Zimmermann, C. Roenneberg, H. Wendorff, T. Holzbach, R. E. Giunta, and H. H. Eckstein, "Early postoperative detection of tissue necrosis in amputation stumps with indocyanine green fluorescence angiography," Vascular and Endovascular Surgery, vol. 44, no. 4, pp. 269-273, 2010.

[145] J. C. Wuestenfeld, J. Herold, U. Niese et al., "Indocyanine green angiography: a new method to quantify collateral flow in mice," Journal of Vascular Surgery, vol. 48, no. 5, pp. 13151321, 2008.

[146] C. Vinegoni, I. Botnaru, E. Aikawa et al., "Indocyanine green enables near-infrared fluorescence imaging of lipidrich, inflamed atherosclerotic plaques," Science Translational Medicine, vol. 3, no. 84, Article ID 84ra45, 2011.

[147] H. Leppikangas, J. J. Tenhunen, L. Lindgren, J. -P. Salenius, and E. Ruokonen, "Effects of levosimendan on indocyanine green plasma disappearance rate and the gastric mucosalarterial $\mathrm{pCO}_{2}$ gradient in abdominal aortic aneurysm surgery," Acta Anaesthesiologica Scandinavica, vol. 52, no. 6, pp. 785-792, 2008.

[148] M. Kikuchi and K. Hosokawa, "Visualized sclerotherapy of varicose veins," Dermatologic Surgery, vol. 36, no. 2, pp. 10501055,2010

[149] W. R. Chen, R. L. Adams, A. K. Higgins, K. E. Bartels, and R. E. Nordquist, "Photothermal effects on murine mammary tumors using indocyanine green and an 808-nm diode laser: an in vivo efficacy study," Cancer Letters, vol. 98, no. 2, pp. 169-173, 1996.

[150] W. R. Chen, W. G. Zhu, J. R. Dynlacht, H. Liu, and R. E. Nordquist, "Long-term tumor resistance induced by laser photo-immunotherapy," International Journal of Cancer, vol. 81, no. 5, pp. 808-812, 1999.

[151] X. Li, G. L. Ferrel, M. C. Guerra et al., "Preliminary safety and efficacy results of laser immunotherapy for the treatment of metastatic breast cancer patients," Photochemical and Photobiological Sciences, vol. 10, no. 5, pp. 817-821, 2011.

[152] R. H. Mellor, A. W. B. Stanton, P. Azarbod, M. D. Sherman, J. R. Levick, and P. S. Mortimer, "Enhanced cutaneous lymphatic network in the forearms of women with postmastectomy oedema," Journal of Vascular Research, vol. 37, no. 6, pp. 501-512, 2000.

[153] H. Lim and N. Soter, Clinical Photomedicine, Marcel Dekker Inc., New York, NY, USA, 1993.

[154] E. Tanaka, H. S. Choi, H. Fujii, M. G. Bawendi, and J. V. Frangioni, "Image-guided oncologic surgery using invisible light: completed pre-clinical development for sentinel lymph node mapping," Annals of Surgical Oncology, vol. 13, no. 12, pp. 1671-1681, 2006.

[155] C. Kim, K. H. Song, F. Gao, and L. V. Wang, "Sentinel lymph nodes and lymphatic vessels: noninvasive dual-modality In vivo mapping by using indocyanine green in ratsvolumetric spectroscopic photoacoustic imaging and planar fluorescence imaging," Radiology, vol. 255, no. 2, pp. 442450, 2010.

[156] N. Ito, M. Fukuta, T. Tokushima, K. Nakai, and S. Ohgi, "Sentinel node navigation surgery using indocyanine green in patients with lung cancer," Surgery Today, vol. 34, no. 7, pp. 581-585, 2004. 
[157] S. Karrer, C. Abels, W. Bäumler, M. Steinbauer, M. Landthaler, and R. M. Szeimies, "Photochemotherapie von kutanen Rektumkarzinom- Metastasen mit Indocyaningrun [photochemotherapy with indocyanine green in cutaneous metastases of rectal carcinoma]," Deutsche medizinische Wochenschrift, vol. 122, no. 37, pp. 1111-1114, 1997.

[158] C. Abels, S. Karrer, W. Bäumler, A. E. Goetz, M. Landthaler, and R.-M. Szeimies, "Indocyanine green and laser light for the treatment of AIDS-associated cutaneous Kaposi's sarcoma," British Journal of Cancer, vol. 77, no. 6, pp. 10211024, 1998.

[159] W. Baumler, C. Abels, S. Karrer et al., "Photo-oxidative killing of human colonic cancer cells using indocyanine green and infrared light," British Journal of Cancer, vol. 80, no. 3-4, pp. 360-363, 1999.

[160] L. M. A. Crane, G. Themelis, H. J. G. Arts et al., "Intraoperative near-infrared fluorescence imaging for sentinel lymph node detection in vulvar cancer: first clinical results," Gynecologic Oncology, vol. 120, no. 2, pp. 291-295, 2011.

[161] S. Inoue, H. Shiina, N. Arichi et al., "Identification of lymphatic pathway involved in the spreading of prostate cancer by fluorescence navigation approach with intraoperatively injected indocyanine green," Journal of the Canadian Urological Association, vol. 5, no. 4, pp. 254-259, 2011.

[162] M. Hutteman, J. S. D. Mieog, J. R. van der Vorst et al., "Randomized, double-blind comparison of indocyanine green with or without albumin premixing for near-infrared fluorescence imaging of sentinel lymph nodes in breast cancer patients," Breast Cancer Research and Treatment, vol. 127, no. 1, pp. 163-170, 2011.

[163] N. Unno, M. Nishiyama, M. Suzuki et al., "A novel method of measuring human lymphatic pumping using indocyanine green fluorescence lymphography," Journal of Vascular Surgery, vol. 52, no. 4, pp. 946-952, 2010.

[164] C. M. Leevy, C. L. Mendenhall, W. Lesko, and M. M. Howard, "Estimation of hepatic blood flow with indocyanine green," The Journal of Clinical Investigation, vol. 41, pp. 1169-1179, 1962.

[165] A. El-Desoky, A. M. Seifalian, M. Cope, D. T. Delpy, and B. R. Davidson, "Experimental study of liver dysfunction evaluated by direct indocyanine green clearance using near infrared spectroscopy," British Journal of Surgery, vol. 86, no. 8, pp. 1005-1011, 1999.

[166] L. R. Jiao, A. A. El-Desoky, A. M. Seifalian, N. Habib, and B. R. Davidson, "Effect of liver blood flow and function on hepatic indocyanine green clearance measured directly in a cirrhotic animal model," British Journal of Surgery, vol. 87, no. 5, pp. 568-574, 2000.

[167] L. R. Jiao, A. A. El-Desoky, A. M. Seifalian, N. Habib, and B. R. Davidson, "Effect of liver blood flow and function on hepatic indocyanine green clearance measured directly in a cirrhotic animal model," British Journal of Surgery, vol. 87, no. 5, pp. 568-574, 2000.

[168] T. Hashimoto, K. Miki, H. Imamura et al., "Sinusoidal perfusion in the veno-occlusive region of living liver donors evaluated by indocyanine green and near-infraredspectroscopy," Liver Transplantation, vol. 14, no. 6, pp. 872-880, 2008.

[169] T. Aoki, D. Yasuda, Y. Shimizu et al., "Image-guided liver mapping using fluorescence navigation system with indocyanine green for anatomical hepatic resection," World Journal of Surgery, vol. 32, no. 8, pp. 1763-1767, 2008.

[170] T. Ishizawa, N. Fukushima, J. Shibahara et al., "Real-time identification of liver cancers by using indocyanine green fluorescent imaging," Cancer, vol. 115, no. 11, pp. 2491-2504, 2009.

[171] K. Gotoh, T. Yamada, O. Ishikawa et al., "A novel imageguided surgery of hepatocellular carcinoma by indocyanine green fluorescence imaging navigation," Journal of Surgical Oncology, vol. 100, no. 1, pp. 75-79, 2009.

[172] H. L. Jun, W. R. Keun, M. C. Kook et al., "Feasibility of laparoscopic sentinel basin dissection for limited resection in early gastric cancer," Journal of Surgical Oncology, vol. 98, no. 5, pp. 331-335, 2008.

[173] H. L. Jun, W. R. Keun, M. -C. Kook et al., "Feasibility of laparoscopic sentinel basin dissection for limited resection in early gastric cancer," Journal of Surgical Oncology, vol. 98, no. 5, pp. 331-335, 2008.

[174] Y.-J. Lee, W. S. Ha, S. T. Park, S. K. Choi, S. C. Hong, and J. W. Park, "Which biopsy method is more suitable between a basin dissection and pick-up biopsy for sentinel nodes in laparoscopic sentinel-node navigation surgery (LSNNS) for gastric cancer?" Journal of Laparoendoscopic and Advanced Surgical Techniques, vol. 18, no. 3, pp. 357-363, 2008.

[175] Y. Tajima, K. Yamazaki, Y. Masuda et al., "Sentinel node mapping guided by indocyanine green fluorescence imaging in gastric cancer," Annals of Surgery, vol. 249, no. 1, pp. 58-62, 2009.

[176] R. A. Cahill, M. Anderson, L. M. Wang, I. Lindsey, C. Cunningham, and N. J. Mortensen, "Near-infrared (NIR) laparoscopy for intraoperative lymphatic road-mapping and sentinel node identification during definitive surgical resection of early-stage colorectal neoplasia," Surgical Endoscopy. In press.

[177] K. Nagata, S. Endo, E. Hidaka, J. I. Tanaka, S. E. Kudo, and A. Shiokawa, "Laparoscopic sentinel node mapping for colorectal cancer using infrared ray laparoscopy," Anticancer Research, vol. 26, no. 3, pp. 2307-2311, 2006.

[178] K. Harada, M. Miwa, T. Fukuyo, S. Watanabe, S. Enosawa, and T. Chiba, "ICG fluorescence endoscope for visualization of the placental vascular network," Minimally Invasive Therapy and Allied Technologies, vol. 18, no. 1, pp. 1-5, 2009.

[179] K. Harada, M. Miwa, T. Fukuyo, S. Watanabe, S. Enosawa, and T. Chiba, "ICG fluorescence endoscope for visualization of the placental vascular network," Minimally Invasive Therapy and Allied Technologies, vol. 18, no. 1, pp. 1-5, 2009.

[180] I. Miyashiro, K. Kishi, M. Yano et al., "Laparoscopic detection of sentinel node in gastric cancer surgery by indocyanine green fluorescence imaging," Surgical Endoscopy and Other Interventional Techniques, vol. 25, no. 5, pp. 1672-1676, 2011.

[181] S.-H. Jeong, Y.-J. Lee, E.-H. Lee et al., "Gastric lymphatic basin dissection for sentinel node biopsy using hybrid natural orifice transluminan endoscopic surgery (NOTES)," Minimally Invasive Therapy \& Allied Technologies, vol. 19, no. 5, pp. 299-303, 2010.

[182] M. J. Tunon, P. Gonzalez, F. Jorquera, A. Llorente, M. Gonzalo-Orden, and J. Gonzalez-Gallego, "Liver blood flow changes during laparoscopic surgery in pigs: a study of hepatic indocyanine green removal," Surgical Endoscopy, vol. 13, no. 7, pp. 668-672, 1999.

[183] K. Murata, K. Shiraki, S. Kamei, K. Takase, and T. Nakano, "Laparoscopic findings in an adult case of Alagille syndrome," Endoscopy, vol. 32, no. 7, pp. 536-538, 2000.

[184] S. Hariharan, Surgical fluorescence imager for visualizing anteriorly placed biliary structures, in-vivo during human cholecystectomy, Ph.D. thesis, The University of Texas, Arlington, Tex, USA, 2008. 
[185] T. Ishizawa, Y. Bandai, M. Ijichi, J. Kaneko, K. Hasegawa, and N. Kokudo, "Fluorescent cholangiography illuminating the biliary tree during laparoscopic cholecystectomy," British Journal of Surgery, vol. 97, no. 9, pp. 1369-1377, 2010.

[186] T. Ishizawa, Y. Bandai, M. Ijichi, J. Kaneko, K. Hasegawa, and N. Kokudo, "Fluorescent cholangiography illuminating the biliary tree during laparoscopic cholecystectomy," British Journal of Surgery, vol. 97, no. 9, pp. 1369-1377, 2010.

[187] A. Matsui, E. Tanaka, H. S. Choi et al., "Real-time intraoperative near-infrared fluorescence identification of the extrahepatic bile ducts using clinically available contrast agents," Surgery, vol. 148, no. 1, pp. 87-95, 2010.

[188] N. Tagaya, M. Shimoda, M. Kato et al., "Intraoperative exploration of biliary anatomy using fluorescence imaging of indocyanine green in experimental and clinical cholecystectomies," Journal of Hepato-Biliary-Pancreatic Sciences, vol. 17, no. 5, pp. 595-600, 2010.

[189] F. Ikeda, K. Yamamoto, S. Fujioka et al., "Laparoscopic findings in primary sclerosing cholangitis," Endoscopy, vol. 33, no. 3, pp. 267-270, 2001.

[190] F. Ikeda, K. Yamamoto, S. Fujioka et al., "Laparoscopic findings in primary sclerosing cholangitis," Endoscopy, vol. 33, no. 3, pp. 267-270, 2001.

[191] H. Mothes, T. Dönicke, R. Friedel, M. Simon, E. Markgraf, and O. Bach, "Indocyanine-green fluorescence video angiography used clinically to evaluate tissue perfusion in microsurgery," Journal of Trauma, vol. 57, no. 5, pp. 10181024, 2004.

[192] L. Prantl, S. Schmitt, S. Geis et al., "Contrast harmonic ultrasound and indocyanine-green fluorescence video angiography for evaluation of dermal and subdermal microcirculation in free parascapular flaps," Clinical Hemorheology and Microcirculation, vol. 38, no. 2, pp. 105-118, 2008.

[193] E. M. Jung, L. Prantl, A. G. Schreyer et al., "New perfusion imaging of tissue transplants with Contrast Harmonic Ultrasound Imaging (CHI) and Magnetic Resonance Imaging (MRI) in comparison with laser-induced Indocyanine Green (ICG) fluorescence angiography," Clinical Hemorheology and Microcirculation, vol. 43, no. 1-2, pp. 19-33, 2009.

[194] H. Mothes, T. Dinkelaker, T. Dönicke, R. Friedel, G. O. Hofmann, and O. Bach, "Outcome prediction in microsurgery by quantitative evaluation of perfusion using ICG fluorescence angiography," Journal of Hand Surgery, vol. 34, no. 2, pp. 238 246, 2009.

[195] M. I. Newman and M. C. Samson, "The application of laserassisted indocyanine green fluorescent dye angiography in microsurgical breast reconstruction," Journal of Reconstructive Microsurgery, vol. 25, no. 1, pp. 21-26, 2009.

[196] A. Matsui, B. T. Lee, J. H. Winer, R. G. Laurence, and J. V. Frangioni, "Predictive capability of near-Infrared fluorescence angiography in submental perforator flap survival," Plastic and Reconstructive Surgery, vol. 126, no. 5, pp. 15181527, 2010.

[197] R. E. Giunta, T. Holzbach, C. Taskov et al., "Prediction of flap necrosis with laser induced indocyanine green fluorescence in a rat model," British Journal of Plastic Surgery, vol. 58, no. 5, pp. 695-701, 2005.

[198] B. T. Lee, A. Matsui, M. Hutteman et al., "Intraoperative near-infrared fluorescence imaging in perforator flap reconstruction: current research and early clinical experience," Journal of Reconstructive Microsurgery, vol. 26, no. 1, pp. 59$65,2010$.

[199] Z.-Z. Jing, S.-M. Chang, M. R. You, and G -R. Yu, "Venous drainage in retrograde island flap: an experimental study using fluorescence tracing technique," Microsurgery, vol. 30, no. 1, pp. 50-54, 2010.

[200] C. Holm, M. Mayr, E. Hofter, U. Dornseifer, and M. Ninkovic, "Assessment of the patency of microvascular anastomoses using microscope-integrated near-infrared angiography: a preliminary study," Microsurgery, vol. 29, no. 7, pp. 509-514, 2009.

[201] I. A. Pestana, B. Coan, D. Erdmann, J. Marcus, L. S. Levin, and M. R. Zenn, "Early experience with fluorescent angiography in free-tissue transfer reconstruction," Plastic and Reconstructive Surgery, vol. 123, no. 4, pp. 1239-1244, 2009.

[202] C. Holm, U. Dornseifer, G. Sturtz, and M. Ninkovic, "Sensitivity and specificity of ICG angiography in free flap reexploration," Journal of Reconstructive Microsurgery, vol. 26, no. 5, pp. 311-316, 2010.

[203] C. Holm, J. Tegeler, M. Mayr, A. Becker, U. J. Pfeiffer, and W. Mühlbauer, "Monitoring free flaps using laser-induced fluorescence of indocyanine green: a preliminary experience," Microsurgery, vol. 22, no. 7, pp. 278-287, 2002.

[204] C. Holm, J. Tegeler, M. Mayr, A. Becker, U. J. Pfeiffer, and W. Muhlbauer, "Monitoring free flaps using laser-induced fluorescence of indocyanine green: a preliminary experience," Microsurgery, vol. 22, no. 7, pp. 278-287, 2002.

[205] K. G. Krishnan, G. Schackert, and R. Steinmeier, "The role of near-infrared angiography in the assessment of postoperative venous congestion in random pattern, pedicled island and free flaps," British Journal of Plastic Surgery, vol. 58, no. 3, pp. 330-338, 2005.

[206] P. Lamby, L. Prantl, S. Gais et al., "Evaluation of the vascular integrity of free flaps based on microcirculation imaging techniques," Clinical Hemorheology and Microcirculation, vol. 39, no. 1-4, pp. 253-263, 2008.

[207] C. S. Betz, S. Zhorzel, H. Schachenmayr et al., "Endoscopic measurements of free-flap perfusion in the head and neck region using red-excited Indocyanine Green: preliminary results," Journal of Plastic, Reconstructive and Aesthetic Surgery, vol. 62, no. 12, pp. 1602-1608, 2009.

[208] C. Holm, M. Mayr, E. Höfter, A. Becker, U. J. Pfeiffer, and W. Mühlbauer, "Intraoperative evaluation of skin-flap viability using laser-induced fluorescence of indocyanine green," British Journal of Plastic Surgery, vol. 55, no. 8, pp. 635-644, 2002.

[209] A. Suzuki, M. Fujiwara, T. Mizukami, and H. Fukamizu, "Delayed distally-based super sural flap: evaluation by indocyanine green fluorescence angiography," Journal of Plastic, Reconstructive and Aesthetic Surgery, vol. 61, no. 4, pp. 467469, 2008.

[210] T. Holzbach, I. Neshkova, D. Vlaskou et al., "Searching for the right timing of surgical delay: angiogenesis, vascular endothelial growth factor and perfusion changes in a skinflap model," Journal of Plastic, Reconstructive and Aesthetic Surgery, vol. 62, no. 11, pp. 1534-1542, 2009.

[211] S. Yamaguchi, F. De Lorenzi, J. Y. Petit et al., "The 'perfusion map' of the unipedicled TRAM flap to reduce postoperative partial necrosis," Annals of Plastic Surgery, vol. 53, no. 3, pp. 205-209, 2004.

[212] C. Holm, M. Mayr, E. Hofter, N. Raab, and M. Ninkovic, "Interindividual variability of the SIEA angiosome: effects on operative strategies in breast reconstruction," Plastic and Reconstructive Surgery, vol. 122, no. 6, pp. 1612-1620, 2008.

[213] J. Quilichini, P. Le Masurier, and T. Guihard, "[Increasing the reliability of SIEA flap using peroperative fluorescent angiography with indocyanine green in breast reconstruction]," Annales de Chirurgie Plastique Esthetique, vol. 55, no. 6, pp. 531-538, 2010 (French). 
[214] B. T. Lee, M. Hutteman, S. Gioux et al., "The fLARE intraoperative near-infrared fluorescence imaging system: a first-inhuman clinical trial in perforator flap breast reconstruction," Plastic and Reconstructive Surgery, vol. 126, no. 5, pp. 1472 1481, 2010.

[215] B. S. Francisco, M. A. Kerr-Valentic, and J. P. Agarwal, "Laserassisted indocyanine green angiography and DIEP breast reconstruction," Plastic and Reconstructive Surgery, vol. 125, no. 3, 2010.

[216] E. Komorowska-Timek and G. C. Gurtner, "Intraoperative perfusion mapping with laser-assisted indocyanine green imaging can predict and prevent complications in immediate breast reconstruction," Plastic and Reconstructive Surgery, vol. 125, no. 4, pp. 1065-1073, 2010.

[217] L.-P. Kamolz, H. Andel, W. Haslik et al., "Indocyanine green video angiographies help to identify burns requiring operation," Burns, vol. 29, no. 8, pp. 785-791, 2003.

[218] L. Devgan, S. Bhat, S. Aylward, and R. J. Spence, "Modalities for the assessment of burn wound depth," Journal of Burns and Wounds, vol. 15, no. 5, p. e2, 2006.

[219] L.-P. Kamolz, H. Andel, T. Auer, G. Meissl, and M. Frey, "Evaluation of skin perfusion by use of indocyanine green video angiography: rational design and planning of trauma surgery," Journal of Trauma, vol. 61, no. 3, pp. 635-641, 2006.

[220] C. Hoffmann, F. Compton, J. H. Schafer et al., "Intraoperative assessment of kidney allgraft perfusion by laser-assisted indocyanine green fluorescence videography," Transplantation Proceedings, vol. 42, no. 5, pp. 1526-1530, 2010.

[221] T. Sawada, M. Solly, J. Kita, M. Shimoda, and K. Kubota, "An alternative tool for intraoperative assessment of renal vasculature after revascularization of a transplanted kidney," American Journal of Surgery, vol. 199, no. 6, pp. e67-e69, 2010.

[222] S. Mizuno and S. Isaji, "Indocyanine green (ICG) fluorescence imaging-guided cholangiography for donor hepatectomy in living donor liver transplantation," American Journal of Transplantation, vol. 10, no. 12, pp. 2725-2726, 2010.

[223] M. Kohl-Bareis, H. Obrig, J. Steinbrink, J. Malak, K. Uludag, and A. Villringer, "Noninvasive monitoring of cerebral blood flow by a dye bolus method: separation of brain from skin and skull signals," Journal of Biomedical Optics, vol. 7, no. 3, pp. 464-470, 2002.

[224] T. S. Leung, I. Tachtsidis, M. Tisdall, M. Smith, D. T. Delpy, and C. E. Elwell, "Theoretical investigation of measuring cerebral blood flow in the adult human head using bolus Indocyanine Green injection and near-infrared spectroscopy," Applied Optics, vol. 46, no. 10, pp. 1604-1614, 2007.

[225] T. Fischer, B. Ebert, J. Voigt et al., "Detection of rheumatoid arthritis using non-specific contrast enhanced fluorescence imaging," Academic Radiology, vol. 17, no. 3, pp. 375-381, 2010.

[226] L. L. Gompels, N. H. Lim, T. Vincent, and E. M. Paleolog, "In vivo optical imaging in arthritis-an enlightening future?" Rheumatology, vol. 49, no. 8, Article ID keq012, pp. 14361446, 2010.

[227] T. Dziekan, C. Weissbach, J. Voigt et al., "Detection of rheumatoid arthritis by evaluation of normalized variances of fluorescence time correlation functions," Journal of Biomedical Optics, vol. 16, no. 7, Article ID 076015, 2011.

[228] K. T. Schomacker, A. Torri, D. R. Sandison, R. L. Sheridan, and N. S. Nishioka, "Biodistribution of indocyanine green in a porcine burn model: light and fluorescence microscopy," Journal of Trauma, vol. 43, no. 5, pp. 813-819, 1997.
[229] H. Habazettl, D. Athanasopoulos, W. M. Kuebler et al., "Near-infrared spectroscopy and indocyanine green derived blood flow index for non-invasive measurement of muscle perfusion during exercise," Journal of Applied Physiology, vol. 108, no. 4, pp. 962-967, 2010.

[230] S. L. Jacques, A. Barofsky, H. Shangguan, S. A. Prahl, and K. W. Gregory, "Laser welding of biomaterials stained with indocyanine green to tissues," in Laser-Tissue Interactions VIII, S. L. Jacques, Ed., vol. SPIE-2975, pp. 54-61, The International Society for Optical Engineering, Bellingham, WA, San Jose, Calif, USA, 1997.

[231] W. Small IV, Thermal and molecular investigation of laser tissue welding, Ph.D. thesis, University of California, Davis, Calif, USA, 1998.

[232] K. Ogan, L. Jacomides, H. Saboorian et al., "Sutureless laparoscopic heminephrectomy using laser tissue soldering," Journal of Endourology, vol. 17, no. 5, pp. 295-300, 2003.

[233] P. Oskoui, I. Stadler, and R. J. Lanzafame, "A preliminary study of laser tissue soldering as arterial wall reinforcement in an acute experimental aneurysm model," Lasers in Surgery and Medicine, vol. 32, no. 5, pp. 346-348, 2003.

[234] G. S. Omar, M. Wilson, and S. P. Nair, "Lethal photosensitization of wound-associated microbes using indocyanine green and near-infrared light," BMC Microbiology, vol. 8, p. 111, 2008.

[235] M. E. Khosroshahi, M. S. Nourbakhsh, S. Saremi, and F. Tabatabaee, "Characterization of skin tissue soldering using diode laser and indocyanine green: In vitro studies," Lasers in Medical Science, vol. 25, no. 2, pp. 207-212, 2010.

[236] F. Rossi, P. Matteini, F. Ratto, L. Menabuoni, I. Lenzetti, and R. Pini, "All Laser' corneal surgery by combination of femtosecond laser ablation and laser tissue welding," in Handbook of Photonics for Biomedical Science, V. V. Tuchin, Ed., pp. 800-810, CRC Press, London, UK, 2010.

[237] H. Ö. Tabakoğlu and M. Gülsoy, "In vivo comparison of near infrared lasers for skin welding," Lasers in Medical Science, vol. 25, no. 3, pp. 411-421, 2010.

[238] J. Shahbazi, H. Marçal, S. Watson, D. Wakefield, M. Sarris, and L. J. R. Foster, "Sutureless sealing of penetrating corneal wounds using a laser-activated thin film adhesive," Lasers in Surgery and Medicine, vol. 43, no. 6, pp. 490-498, 2011.

[239] I. Y. Yanina, V. A. Bochko, J. T. Alander, and V. V. Tuchin, "Optical image analysis of fat cells for indocyanine green mediated near-infrared laser treatment," Laser Physics Letters, vol. 8, no. 9, pp. 684-690, 2011.

[240] Y. P. Krespi and V. Kizhner, "Phototherapy for chronic rhinosinusitis," Lasers in Surgery and Medicine, vol. 43, no. 3, pp. 187-191, 2011.

[241] W. Hongcharu, C. R. Taylor, Y. Chang, D. Aghassi, K. Suthamjariya, and R. R. Anderson, "Topical ALA-photodynamic therapy for the treatment of acne vulgaris," Journal of Investigative Dermatology, vol. 115, no. 2, pp. 183-192, 2000.

[242] V. V. Tuchin, E. A. Genina, A. N. Bashkatov, G. V. Simonenko, O. D. Odoevskaya, and G. B. Altshuler, "A pilot study of ICG laser therapy of acne vulgaris: photodynamic and photothermolysis treatment," Lasers in Surgery and Medicine, vol. 33, no. 5, pp. 296-310, 2003.

[243] E. A. Genina, A. N. Bashkatov, G. V. Simonenko, O. D. Odoevskaya, V. V. Tuchin, and G. B. Altshuler, "Low-intensity indocyanine-green laser phototherapy of acne vulgaris: pilot study," Journal of Biomedical Optics, vol. 9, no. 4, pp. 828-834, 2004.

[244] N. Price, M. R. Gottfried, E. Clary et al., "Safety and efficacy of India ink and indocyanine green as colonic tattooing 
agents," Gastrointestinal Endoscopy, vol. 51, no. 4, pp. 438442, 2000.

[245] E. A. Genina, A. N. Bashkatov, Y. P. Sinichkin et al., "In vitro and in vivo study of dye diffusion into the human skin and hair follicles," Journal of Biomedical Optics, vol. 7, no. 3, pp. 471-477, 2002.

[246] N. Miyoshi, M. Ohue, S. Noura et al., "Surgical usefulness of indocyanine green as an alternative to India ink for endoscopic marking," Surgical Endoscopy and Other Interventional Techniques, vol. 23, no. 2, pp. 347-351, 2009.

[247] M. Watanabe, A. Tsunoda, K. Narita, M. Kusano, and M. Miwa, "Colonic tattooing using fluorescence imaging with light-emitting diode-activated indocyanine green: a feasibility study," Surgery Today, vol. 39, no. 3, pp. 214-218, 2009.

[248] S. E. Boddington, T. D. Henning, P. Jha et al., "Labeling human embryonic stem cell-derived cardiomyocytes with indocyanine green for noninvasive tracking with optical imaging: an FDA-compatible alternative to firefly luciferase," Cell Transplantation, vol. 19, no. 1, pp. 55-65, 2010.

[249] K. Kasuya, K. Sugimoto, B. Kyo et al., "Ultrasonographyguided hepatic tumor resection using a real-time virtual sonography with indocyanine green navigation (with videos)," Journal of Hepato-Biliary-Pancreatic Sciences, vol. 18, no. 3, pp. 380-385, 2011.

[250] R. Esenaliev, A. Oraevsky, S. Rastegar, C. Frederickson, and M. Motamedi, "Mechanism of dye-enhanced pulsed laser ablation of hard tissues: implications for dentistry," IEEE Journal on Selected Topics in Quantum Electronics, vol. 2, no. 4, pp. 836-846, 1996.

[251] T. Kitai, M. Kawashima, H. Fujii, S. Mashima, and Y. Shimahara, "Indocyanine green fluorescence monitoring of perineal wound contamination in abdominoperineal resection: a preliminary report," Surgery Today, vol. 41, no. 8, pp. 1037-1040, 2011.

[252] W. Li, D. Wang, J. Qin et al., "Generation of functional hepatocytes from mouse induced pluripotent stem cells," Journal of Cellular Physiology, vol. 222, no. 3, pp. 492-501, 2010.

[253] M. Zhou, P. Li, L. Tan, S. Qu, Q. L. Ying, and H. Song, "Differentiation of mouse embryonic stem cells into hepatocytes induced by a combination of cytokines and sodium butyrate," Journal of Cellular Biochemistry, vol. 109, no. 3, pp. 606-614, 2010.

[254] K. Koivuporras, Enhancing a neurosurgical imaging system with a PCbased video processing solution, M.S. thesis, University of Vaasa, Ostrobothnia, Finland, 2011.

[255] J. Oda, Y. Kato, S. F. Chen et al., "Intraoperative near-infrared indocyanine green-videoangiography (ICG-VA) and graphic analysis of fluorescence intensity in cerebral aneurysm surgery," Journal of Clinical Neuroscience, vol. 18, no. 8, pp. 1097-1100, 2011.

[256] E. M. C. Hillman and A. Moore, "All-optical anatomical coregistration for molecular imaging of small animals using dynamic contrast," Nature Photonics, vol. 1, no. 9, pp. 526530, 2007.

[257] V. P. Zharov, E. I. Galanzha, E. V. Shashkov, J. W. Kim, N. G. Khlebtsov, and V. V. Tuchin, "Photoacoustic flow cytometry: principle and application for real-time detection of circulating single nanoparticles, pathogens, and contrast dyes In vivo," Journal of Biomedical Optics, vol. 12, no. 5, Article ID 051503, 2007.

[258] Y. Kang, J. Lee, K. Kwon, and C. Choi, "Assessment of peripheral tissue perfusion by optical dynamic fluorescence imaging and nonlinear regression modeling," in Photonic
Therapeutics and Diagnostics VI, N. Kollias, Ed., vol. SPIE7548, pp. 7548L-75483L, The International Society for Optical Engineering, Bellingham, Wash, USA, 2010.

[259] M. Diop, K. Verdecchia, T.-Y. Lee, and K. St. Lawrence, "Calibration of diffuse correlation spectroscopy with a timeresolved nearinfrared technique to yield absolute cerebral blood flow measurements," Biomedical Optics Express, vol. 2, no. 7, pp. 2068-2082, 2011.

[260] P. Ferroli, P. Nakaji, F. Acerbi, E. Albanese, and G. Broggi, "Indocyanine green (ICG) temporary clipping test to assess collateral circulation before venous sacrifice," World Neurosurgery, vol. 75, no. 1, pp. 122-125, 2011.

[261] X. Liu, F. Liu, and J. Bai, "A linear correction for principal component analysis of dynamic fluorescence diffuse optical tomography images," IEEE Transactions on Biomedical Engineering, vol. 58, no. 6, pp. 1602-1611, 2011.

[262] A. Rodríguez-Hernández and M. T. Lawton, "Flash fluorescence with icg videoangiography to identify the recipient artery for bypass with distal middle cerebral artery aneurysms: operative technique," Neurosurgery. In press.

[263] V. Ntziachristos, A. G. Yodh, M. Schnall, and B. Chance, "Concurrent MRI and diffuse optical tomography of breast after indocyanine green enhancement," Proceedings of the National Academy of Sciences of the United States of America, vol. 97, no. 6, pp. 2767-2772, 2000.

[264] B. S. Sandanaraj, R. Kneuer, and N. Beckmann, "Optical and magnetic resonance imaging as complementary modalities in drug discovery," Future Medicinal Chemistry, vol. 2, no. 3, pp. 317-337, 2010.

[265] A. Ashokan, P. Chandran, A. R. Sadanandan et al., "Development and haematotoxicological evaluation of doped hydroxyapatite based multimodal nanocontrast agent for nearinfrared, magnetic resonance and X-ray contrast imaging," Nanotoxicology. In press.

[266] Y. Lin, M. T. Ghijsen, H. Gao, N. Liu, O. Nalcioglu, and G. Gulsen, "A photo-multiplier tube-based hybrid MRI and frequency domain fluorescence tomography system for small animal imaging," Physics in Medicine and Biology, vol. 56, no. 15, pp. 4731-4747, 2011.

[267] X. Liu, D. Wang, F. Liu, and J. Bai, "Principal component analysis of dynamic fluorescence diffuse optical tomography images," Optics Express, vol. 18, no. 6, pp. 6300-6314, 2010.

[268] M. Goetz, I. Deris, M. Vieth et al., "Near-infrared confocal imaging during mini-laparoscopy: a novel rigid endomicroscope with increased imaging plane depth," Journal of Hepatology, vol. 53, no. 1, pp. 84-90, 2010.

[269] J. S.D. Mieog, S. L. Troyan, M. Hutteman et al., "Toward optimization of imaging system and lymphatic tracer for near-infrared fluorescent sentinel lymph node mapping in breast cancer," Annals of Surgical Oncology, vol. 18, no. 9, pp. 2483-2491, 2011.

[270] F. Tam, G. P. Goodrich, B. R. Johnson, and N. J. Halas, "Plasmonic enhancement of molecular fluorescence," Nano Letters, vol. 7, no. 2, pp. 496-501, 2007.

[271] E. S. Tuchina, V. V. Tuchin, B. N. Khlebtsov, and N. G. Khlebtsov, "Phototoxic effect of conjugates of plasmonresonance nanoparticles with indocyanine green dye on Staphylococcus aureus induced by IR laser radiation," Quantum Electronics, vol. 41, no. 4, pp. 354-359, 2011.

[272] T. Buckle, A. C. van Leeuwen, P. T. K. Chin et al., "A self-assembled multimodal complex for combined pre- and intraoperative imaging of the sentinel lymph node," Nanotechnology, vol. 21, no. 35, Article ID 355101, 2010. 
[273] Y. Ashitate, A. Stockdale, H. S. Choi, R. G. Laurence, and J. V. Frangioni, "Real-time simultaneous near-infrared fluorescence imaging of bile duct and arterial anatomy," Journal of Surgical Research. In press.

[274] S. Tobis, J. Knopf, C. Silvers et al., "Near infrared fluorescence imaging with robotic assisted laparoscopic partial nephrectomy: initial clinical experience for renal cortical tumors," Journal of Urology, vol. 186, no. 1, pp. 47-52, 2011.

[275] J. T. Alander, "A review of indocyanine green contrast agent in surgery," in Proceedings of the 14th International Conference in Near Infrared Spectroscopy, S. Sarangwong, S. Kasemsumran, W. Thanapase, and P. Williams, Eds., pp. 615-619, IM Publications LLP, Bangkok, Thailand, November 2009. 

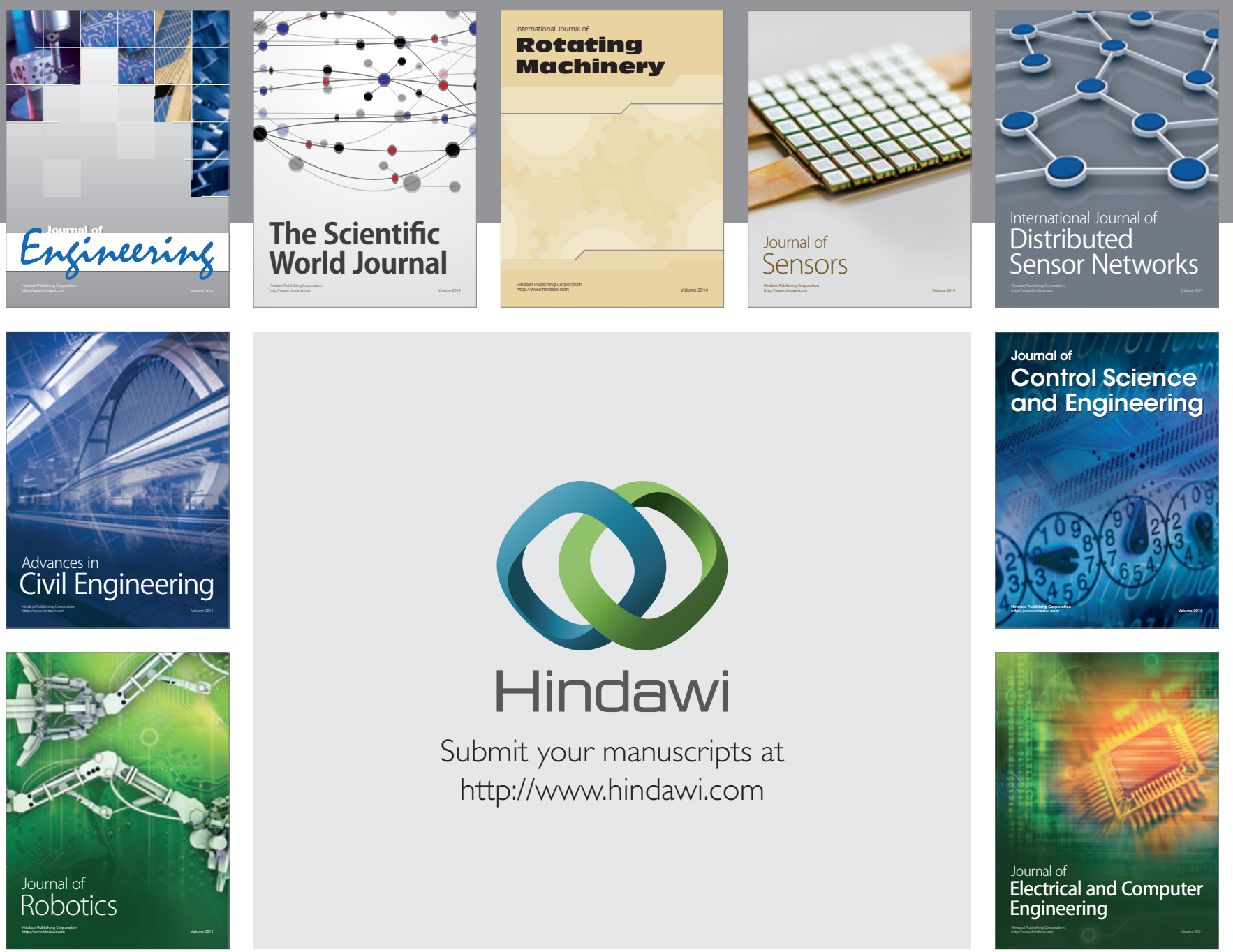

Submit your manuscripts at

http://www.hindawi.com
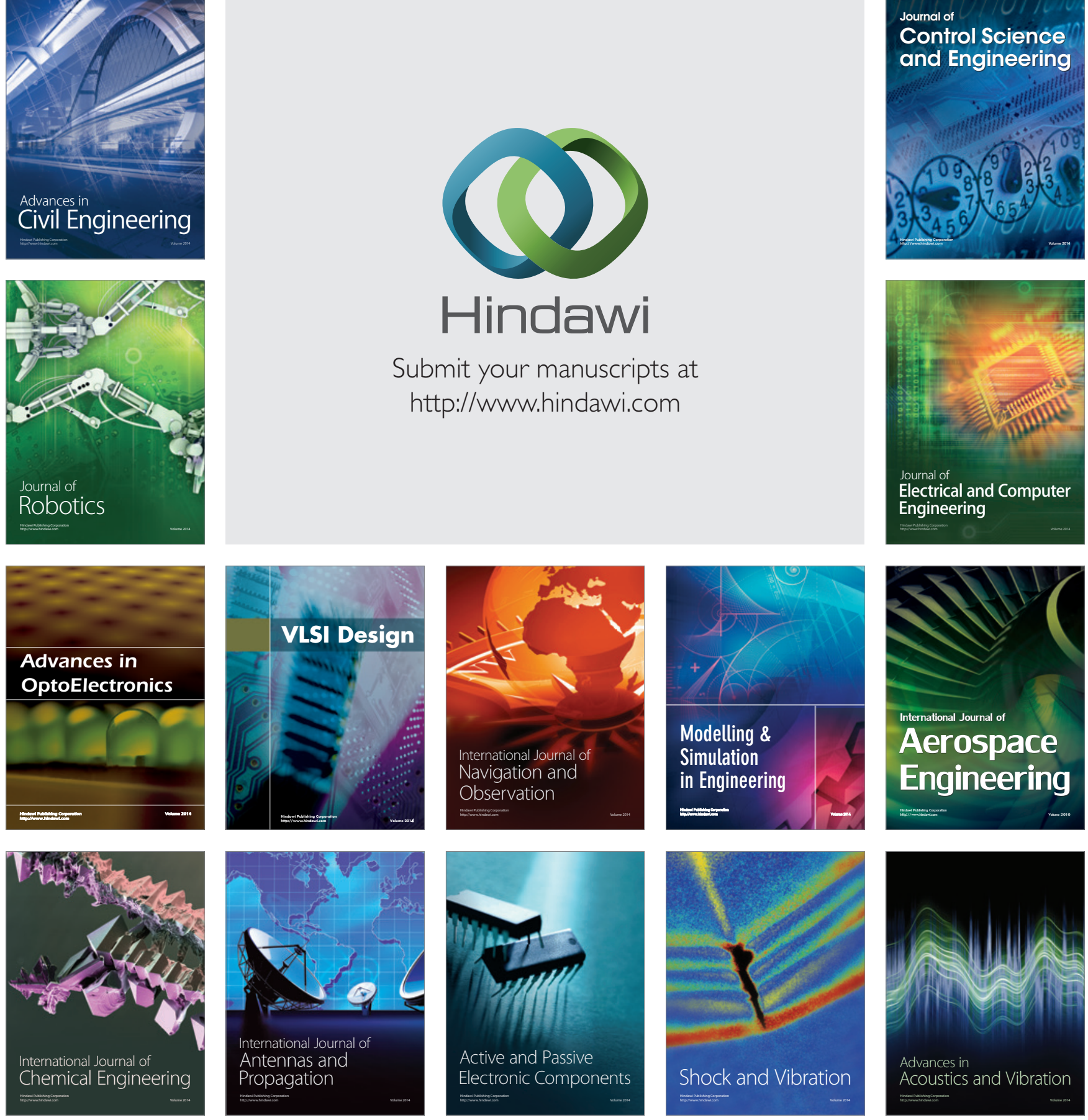